\title{
Quantum Entanglement and Geometry of Determinantal Varieties
}

\author{
Hao Chen \\ Department of Mathematics \\ Zhongshan University \\ Guangzhou,Guangdong 510275 \\ People's Republic of China \\ e-mail: mcsch@zsu.edu.cn
}

April, 2003

\begin{abstract}
Quantum entanglement was first recognized as a feature of quantum mechanics in the famous paper of Einstein, Podolsky and Rosen [18]. Recently it has been realized that quantum entanglement is a key ingredient in quantum computation, quantum communication and quantum cryptography $([16],[17],[6])$. In this paper, we introduce algebraic sets, which are determinantal varieties in the complex projective spaces or the products of complex projective spaces, for the mixed states in bipartite or multipartite quantum systems as their invariants under local unitary transformations. These invariants are naturally arised from the physical consideration of measuring mixed states by separable pure states. In this way algebraic geometry and complex differential geometry of these algebraic sets turn to be powerful tools for the understanding of quantum enatanglement. Our construction has applications in the following important topics in quantum information theory: 1) separability criterion, it is proved the algebraic sets have to be the sum of the linear subspaces if the mixed states are separable; 2) lower bound of Schmidt numbers, that is, generic low rank bipartite mixed states are entangled in many degrees of freedom; 3) simulation of Hamiltonians, it is proved the simulation of semi-positive Hamiltonians of the same rank implies the projective isomorphisms of the corresponding algebraic sets; 4) construction of bound enatanglement, examples of the entangled mixed states which are invariant under partial transpositions (thus PPT bound entanglement) are constructed systematically from our new separability criterion. On the other hand many examples of entangled mixed states with rich algebraic-geometric structure in their associated determinantal varieties are constructed and studied from this point of view.
\end{abstract}




\section{Introduction}

A bipartite pure quantum state $|\psi\rangle \in H=H_{A}^{m} \otimes H_{B}^{n}$, where $H_{A}, H_{B}$ are finite dimensional Hilbert spaces and the tensor inner product is used on $H$, is called entangled if it cannot be written as $|\psi\rangle=\left|\psi_{A}\right\rangle \otimes\left|\psi_{B}\right\rangle$ for some $\left|\psi_{A}\right\rangle \in H_{A}$ and $\left|\psi_{B}\right\rangle \in H_{B}$. A mixed state (or a density matrix) $\rho$, which is a positive semidefinite operator on $H$ with trace 1 , is called entangled if it cannot be written as

$$
\rho=\Sigma_{i} p_{i} P_{\left|\psi_{i}^{A}\right\rangle} \otimes P_{\left|\psi_{i}^{B}\right\rangle}
$$

, for some set of states $\left|\psi_{i}^{A}\right\rangle \in H_{A},\left|\psi_{i}^{B}\right\rangle \in H_{B}$ and $p_{i} \geq 0$. Here $P_{v}$ for a state (unit vector) $v$ means the (rank 1 ) projection operator to the vector $v$. If the mixed state $\rho$ can be written in the form of (1), it is called separable (see $[28],[39][42])$.

For the mixed state $\rho$ on $H=H_{A} \otimes H_{B}$,we have the following partial transposition $\rho^{P T}$ and the partial trace $\operatorname{tr}_{B}(\rho)$ on $H_{A}^{m}\left(\operatorname{tr}_{A}(\rho)\right.$ on $H_{B}^{n}$ can be defined similarly) defined as follows.

$$
\begin{gathered}
\left\langle i j\left|\rho^{P T}\right| k l\right\rangle=\langle i l|\rho| k j\rangle \\
\left\langle i\left|\operatorname{tr}_{B}(\rho)\right| k\right\rangle=\Sigma_{j}\langle i j|\rho| k j\rangle
\end{gathered}
$$

, where $\{|1\rangle, \ldots,|m\rangle\},\{|1\rangle, \ldots,|n\rangle\},\{|11\rangle, \ldots|1 n\rangle, \ldots|m 1\rangle, \ldots,|m n\rangle\}$ are the standard orthogonal basis of $H_{A}, H_{B}, H$ respectively. It is easy to check that the definition is not dependent on the special basis ([28],[39],[42]).

For multipartite quantum systems, there are similar definitions of entangled and separable mixed states (see [28],[39],[42]). We restrict ourselves to the case of the tripartite case. Let $\rho$ be a mixed state in $H=H_{A} \otimes H_{B} \otimes H_{C} . \rho$ is called separable if it can be written as

$$
\rho=\Sigma_{i} p_{i} P_{\left|\psi_{i}^{A}\right\rangle} \otimes P_{\left|\psi_{i}^{B}\right\rangle} \otimes P_{\left|\psi_{i}^{C}\right\rangle}
$$

, for some set of states $\left|\psi_{i}^{A}\right\rangle \in H_{A},\left|\psi_{i}^{B}\right\rangle \in H_{B},\left|\psi_{i}^{C}\right\rangle \in H_{C}$ and $p_{i} \geq 0$. If the mixed state $\rho$ cannot be written in the form of (3), it is called entangled. Sometimes we also consider the separability relative to the cut A:BC (B:AC etc.), that means $\rho$ is considered as a mixed state in the bipartite quantum system $H=H_{A} \otimes\left(H_{B} \otimes H_{C}\right)$.

For a n-party quantum system $H=H_{A_{1}}^{m_{1}} \otimes \ldots \otimes H_{A_{n}}^{m_{n}}$, local unitary transformations (acting on a mixed state $\rho$ by $U \rho U^{\dagger}$, where $\dagger$ is the adjoint.) are those unitary transformations of the form $U=U_{A_{1}} \otimes \ldots \otimes U_{A_{n}}$, where $U_{A_{i}}$ is a unitary transformation on $H_{A_{i}}^{m_{i}}$ for $i=1, \ldots, n$. We can check that all eigenvalues (spectra) of $\rho$ (global spectra) and $\operatorname{tr}_{A_{i_{1}} \ldots A_{i_{l}}}(\rho)$ of mixed states 
$\rho$, where $i_{1}, \ldots, i_{l} \in\{1, \ldots, n\}$, (local spectra) are invariant under local unitary transformations, and the invariants in the examples of [33] and [34] are more or less spectra-involved. It is clear that separability (or being entangled) is an invariant property under local unitary transformations. For a mixed state $\rho$ , to judge whether it is entangled or separable and decide its entangled class (i.e. the equivalent class of all entangled (or separable) mixed states which are equivalent to $\rho$ by local unitary transformations) is a fundamental problem in the study of quantum entanglement ([28],[42]). Thus for the purpose to quantify entanglement, any good measure of entanglement must be invariant under local transformations ([33],[34],[28],[42]). Another important concept is the distillable mixed state, which means that some singlets can be extracted from it by local operations and classical communication (LOCC) (see [28]). A mixed state $\rho$ in $H$ is distillable if and only if for some $t$, there exists projections $P_{A}: H_{A}^{\otimes t} \rightarrow H^{2}$ and $P_{B}: H_{B}^{\otimes t} \rightarrow H^{2}$, where $H^{2}$ is a 2 dimensional Hilbert space, such that the mixed state $\left(P_{A} \otimes P_{B}\right) \rho^{\otimes t}\left(P_{A} \otimes P_{B}\right)^{\dagger}$ is a entanlged state in $H^{2} \otimes H^{2}$ (see [28]). A mixed state which cannot be distilled is called bounded entangled mixed state.

The phonomenon of quantum entanglement lies at the heart of quantum mechanics since the famous Einstein, Podolsky and Rosen [18] paper (see [5],[28],[39]). Its importance lies not only in philosophical consideration of the nature of quantum theory, but also in applications where it has emerged recently that quantum entanglement is the key ingredient in quantum computation [16] and communication [6] and plays an important role in cryptography ([17]). These new applications of quantum entanglement have stimulated tremendous studies of quantum entanglements of both pure and mixed states from both theoretical and experimental view, for surveys we refer to [5],[28],[32],[39] and [42].

To find good necessary condition of separability (separability criterion) is a fundamental problem in the study of quantum entanglement ([28],[42]). Belltype inequalities ([28]) and entropy criterion ([28]) are well-known numerical criterion of separable states. In 1996, Peres [38] gave a striking simple criterion which asserts that a separable mixed state $\rho$ necessarily has (semi) positive partial transposition (PPT), which has been proved by Horodeckis ([24]) also a sufficient condition of separability in $2 \times 2$ and $2 \times 3$ systems. The significance of PPT property is also reflected in the facts that PPT mixed states satisfy Bell inequalities ([44]) and cannot be distilled ([28],[26]), thus the first several examples of the PPT entangled mixed states ([25]) indicated that the new phenomenon that there is bound entanglement in nature ([26]). These examples were constructed from the so-called range criterion of P.Horodecki (see [25],[28]). However constructing PPT entangled mixed states (thus bound entanglement) is exceedingly difficult task $([7])$, and the only known systematic way of such construction is the context of unextendible product base (UPB) in [7], which works in both bipartite and multipartite case and is also based on P.Horodecki's range criterion. The most recent disorder criterion of separability in [37], which is stronger than entropy criterion, was proved by the mathematics of majorization. 
It has been realized that the entanglement of tripartite pure quantum states is not a trivial extension of the entanglement of bipartite pure quantum states ([21]). Recently C.H.Bennett, etc., [8] studied the exact and asymptotic entanglement measure of multipartite pure states, which showed essential difference to that of bipartite pure states. On the other hand Acin, etc., [2] proved a generalization of Schmidt decomposition for pure triqubit states, which seems impossible to be generalized to arbitrary multipartite case . Basically, the understanding of multipartite quantum entanglement for both pure and mixed states, is much less advanced.

It is clear that any separability criterion for bipartite mixed states, such as Peres PPT criterion [38] and Horodecki range criterion ([25],[28]), can be applied to multipartite mixed states for their separability under various cuts. For example, from Peres PPT criterion, a separable multipartite mixed state necessarily has all its partial transpositions semi-positive. In [27], Horodeckis studied the separability criterion of multipartite mixed states by linear maps. Classification of triqubit mixed states inspired by Acin, etc., [2] was studied in [3].

There have been many interesting works [11],[12],[31],[35] and [43] for understanding quantum entanglemment and related problems from the view of Representation Theory and Topology.

The physical motivation of this paper is as follows. We consider the following situation. Alice and Bob share a bipartite quantum system $H_{A}^{m} \otimes H_{B}^{n}$, and they have a mixed state $\rho$. Now they want to understand the entanglement properties of $\rho$. It is certain that they can prepare any separable pure state $\left|\phi_{1}\right\rangle \otimes\left|\phi_{2}\right\rangle$ seaprately. Now they measure $\rho$ with this separable pure state, the expectation value is $\left\langle\phi_{1} \otimes \phi_{2}|\rho| \phi_{1} \otimes \phi_{2}\right\rangle$. If Alice's pure state $\left|\phi_{1}\right\rangle$ is fixed, then $\left\langle\phi_{1} \otimes \phi_{2}|\rho| \phi_{1} \otimes \phi_{2}\right\rangle$ is a Hermitian bilinear form on Bob's pure states (i.e., on $\left.H_{B}^{n}\right)$. We denote this bilinear form by $\left\langle\phi_{1}|\rho| \phi_{1}\right\rangle$. Intuitively the degenerating locus $V_{A}^{k}(\rho)=\left\{\left|\phi_{1}\right\rangle \in P\left(H_{A}^{m}\right): \operatorname{rank}\left(\left\langle\phi_{1}|\rho| \phi_{1}\right\rangle\right) \leq k\right\}$, where $P\left(H_{A}^{m}\right)$ is the projective space of all pure states in $H_{A}^{m}$, should contain the physical information of $\rho$ and it is almost obvious that these degenerating locus are invariant under local unitary transformations. In a multipartite quantum system, a similar consideration leads to some Hermitian bilinear forms on some of its parts and similarly we can consider the degenerating locus of these Hermitian bilinear forms. We prove that these degenerating locus are algebraic sets, which are determinantal varieties, for the mixed states in both bipartite and multipartite quantum systems. They have the following properties:

1) When we apply local unitary transformations on the mixed state the corresponding algebraic sets are changed by local (unitary) linear transformations, and thus these invariants can be used to distinguish inequivalent mixed states under local unitary transformations; 
2) The algebraic sets have to be linear (the sum of some linear subspaces) if the mixed state is separable, and thus we give a new separability criterion;

3) The algebraic sets are independent of eigenvalues and only measure the positions of eigenvectors of the mixed states;

4) These algebraic sets can be calculated easily.

From our construction here, we establish a connection between Quantum Entanglement and Algebraic Geometry. Actually from our results below, we can see that if the Fubini-Study metric of the projective complex space is used, the metric properties of these algebraic sets are also preserved when local unitary transformations are applied on the mixed state. Hence we establish a connection between Quantum Entanglement and both the Algebraic Geometry and complex Differential Geometry of these algebraic sets. Any algebraic-geometric or complex differential geometric invariant of the algebraic set of the mixed state is an invariant of the mixed state under local unitary transformations.

The determinantal varieties ([23] Lecture 9 and [4] Cha.II) have been studied from different motivations such as geometry of curves ([4],[19]), geometry of determinantal varieties([20]), Hodge theory $([22])$, commutative algebra ([15]) and even combinatorics ([1]). It is interesting to see that it can be useful even in quantum information theory. We refer to the books [23] and [4] for the standard facts about determinantal varieties.

The paper is organized as follows. We define the algebraic sets of the mixed states and prove their basic properties including the separability criteria based on these algebraic sets in section 2 . In section 3 , we indicate briefly how numerical invariants of the bipartite or multipartite mixed states under local unitary transformations can be derived from these algebraic sets. As an easiest example, Schmidt rank of a pure state in bipartite quantum systems, a classical concept in quantum entanglement, is showed to be the codimention of the algebraic set. From our invariants, a LOWER bound of Schmidt numbers of mixed states (defined in [41]) is given (Theorem 4), which implies that the "generic" rank $m$ mixed states on $H_{A}^{m} \otimes H_{B}^{m}$ have relatively high Schmidt numbers. Many examples of entangled mixed states corresponding to the famous determinantal varieties in algebraic geometry, such as Segre varieties, rational normal scrolls and generic determinantal varieties are constructed in section 4 . We also show that a well-known theorem of D.Eisenbud ([20]) can help us to construct many entangled bipartite mixed states of low ranks. In section 5 we introduce the generalized Smolin states, which is a natural extension of Smolin's physical construction [40] from algebraic-geometric view. In section 6 and 7, it is proved that these algebraic sets are nonempty for low rank mixed states, and indicate how a finer result with the same idea (Theorem 9') can be potentially used to treat the entanglement properties of high rank mixed states. Based on the al- 
gebraic sets introduced in section 2 , a necessary condition about the simulating Hamiltonians efficiently using local unitary transformations is given in section 8. In section 9 , we give a continuous family of bipartite mixed states, tripartite pure states and bipartite Hamiltonians with the property that the eigenvalues (spectra) of them and their partial traces are constant, however their entanglement properties are distinct. This offers strong evidences that it is hopeless to characterize the entanglement properties by only using eigenvalue spectra. In section 10, we illustrate by an explicit example that our separability crieterion can be used to construct PPT entangled mixed states (thus bound entanglement) systematically.

\section{Invariants and separability criteria}

Now we use the coordinate form of the physical consideration of degenerating locus described in the introduction. Let $H=H_{A}^{m} \otimes H_{B}^{n},\{|i j\rangle\}$, where, $i=1, \ldots, m$ and $j=1, \ldots, n$, be the standard basis and $\rho$ be a mixed state on $H$. We represent the matrix of $\rho$ in the basis $\{|11\rangle, \ldots|1 n\rangle, \ldots,|m 1\rangle, \ldots,|m n\rangle\}$, and consider $\rho$ as a blocked matrix $\rho=\left(\rho_{i j}\right)_{1 \leq i \leq m, 1 \leq j \leq m}$ with each block $\rho_{i j}$ a $n \times n$ matrix corresponding to the $|i 1\rangle, \ldots,|i n\rangle$ rows and the $|j 1\rangle, \ldots,|j n\rangle$ columns. For any pure state $\left|\phi_{1}\right\rangle=r_{1}|1\rangle+\ldots+r_{m}|m\rangle \in P\left(H_{A}^{m}\right)$ the matrix of the Hermitian linear form $\left\langle\phi_{1}|\rho| \phi_{1}\right\rangle$ with the basis $|1\rangle, \ldots,|n\rangle$ is $\Sigma_{i, j} r_{i} r_{j}^{\dagger} \rho_{i j}$. Thus the degenerating locus are as follows.

Definition 1. We define

$$
V_{A}^{k}(\rho)=\left\{\left(r_{1}, \ldots, r_{m}\right) \in C P^{m-1}: \operatorname{rank}\left(\Sigma_{i, j} r_{i} r_{j}^{\dagger} \rho_{i j}\right) \leq k\right\}
$$

where $k=0,1, \ldots, n-1$.Similarly $V_{B}^{k}(\rho) \subseteq C P^{n-1}$, where $k=0,1, \ldots, m-1$, can be defined.

Example 1. Let $\rho=\frac{1}{m n} I_{m n}$, the maximally mixed state, we easily have $V_{A}^{k}(\rho)=\left\{\left(r_{1}, \ldots, r_{n}\right): \operatorname{rank}\left(\Sigma r_{i} r_{i}^{\dagger} I_{n}\right) \leq k\right\}=\emptyset$ for $k=0,1, \ldots, n-1$.

Example 2. Let $H=H_{A}^{2} \otimes H_{B}^{n}, T_{1}, T_{2}$ be $2 n \times n$ matrices of rank $n-1$ such that the $n \times(2 n)$ matrix $\left(T_{1}, T_{2}\right)$ has rank $n$. Let $T^{\prime}$ be a $2 n \times 2 n$ matrix with 11 block $T_{1}, 22$ block $T_{2}, 12$ and 21 blocks 0 . Its rows correspond to the standard base $|11\rangle, \ldots,|1 n\rangle,|21\rangle, \ldots,|2 n\rangle$ of $H$. Let $\rho=\frac{1}{D} T T^{\dagger}$ (where $D$ is a normalizing constant) be a mixed state on $H$. It is easy to check that $\rho$ is of rank $2 n-2$ and $V_{A}^{n-1}(\rho)=\left\{\left(r_{1}, r_{2}\right): r_{1} r_{2}=0\right\}$.

Let $H=H_{A}^{m} \otimes H_{B}^{n} \otimes H_{C}^{l}$. The standard orthogonal basis is of $H$ is $|i j k\rangle$, where, $i=1, \ldots, m, j=1, \ldots, n$ and $k=1, \ldots, l$, and $\rho$ is a mixed state on $H$. We represent the matrix of $\rho$ in the base $\{|111\rangle, \ldots|11 l\rangle, \ldots,|m n 1\rangle, \ldots,|m n l\rangle\}$ as 
$\rho=\left(\rho_{i j, i^{\prime} j^{\prime}}\right)_{1 \leq i, i^{\prime} \leq m, 1 \leq j, j^{\prime} \leq n}$, and $\rho_{i j, i^{\prime} j^{\prime}}$ is a $l \times l$ matrix. Consider $H$ as a bipartite system as $H=\left(H_{A}^{m} \otimes H_{B}^{n}\right) \otimes H_{C}^{l}$, then we have $V_{A B}^{k}(\rho)=\left\{\left(r_{11}, \ldots, r_{m n}\right) \in\right.$ $\left.C P^{m n-1}: \operatorname{rank}\left(\Sigma r_{i j} r_{i^{\prime} j^{\prime}}^{\dagger} \rho_{i j, i^{\prime} j^{\prime}}\right) \leq k\right\}$ defined as above. This set is actually the degenerating locus of the Hermitian bilinear form $\left\langle\phi_{12}|\rho| \phi_{12}\right\rangle$ on $H_{C}^{l}$ for the given pure state $\left|\phi_{12}\right\rangle=\Sigma_{i, j}^{m, n} r_{i j}|i j\rangle \in P\left(H_{A}^{m} \otimes H_{B}^{n}\right)$. When the finer cut A:B:C is considered, it is natural to take $\left|\phi_{12}\right\rangle$ as a separable pure state $\left|\phi_{12}\right\rangle=\left|\phi_{1}\right\rangle \otimes\left|\phi_{2}\right\rangle$, ie., there exist $\left|\phi_{1}\right\rangle=\Sigma_{i} r_{i}^{1}|i\rangle \in P\left(H_{A}^{m}\right),\left|\phi_{2}\right\rangle=\Sigma_{j} r_{j}^{2}|j\rangle \in P\left(H_{B}^{n}\right)$ such that $r_{i j}=r_{i}^{1} r_{j}^{2}$. In this way the tripartite mixed state $\rho$ is measured by tripartite separable pure states $\left|\phi_{1}\right\rangle \otimes\left|\phi_{2}\right\rangle \otimes\left|\phi_{3}\right\rangle$. Thus it is natural we define $V_{A: B}^{k}(\rho)$ as follows. It is the degenerating locus of the bilinear form $\left\langle\phi_{1} \otimes \phi_{2}|\rho| \phi_{1} \otimes \phi_{2}\right\rangle$ on $H_{C}^{l}$.

Definition 2. Let $\phi: C P^{m-1} \times C P^{n-1} \rightarrow C P^{m n-1}$ be the mapping defined by

$$
\phi\left(r_{1}^{1}, \ldots r_{m}^{1}, r_{1}^{2}, \ldots, r_{n}^{2}\right)=\left(r_{1}^{1} r_{1}^{2}, \ldots, r_{i}^{1} r_{j}^{2}, \ldots r_{m}^{1} r_{n}^{2}\right)
$$

(i.e., $r_{i j}=r_{i}^{1} r_{j}^{2}$ is introduced.)

Then $V_{A: B}^{k}(\rho)$ is defined as the preimage $\phi^{-1}\left(V_{A B}^{k}(\rho)\right)$.

Similarly $V_{B: C}^{k}(\rho), V_{A: C}^{k}(\rho)$ can be defined. In the following statement we just state the result for $V_{A: B}^{k}(\rho)$. The conclusion holds similarly for other $V^{\prime} s$.

For the mixed state $\rho$ in the multipartite system $H=H_{A_{1}}^{m_{1}} \otimes \cdots \otimes H_{A_{k}}^{m_{k}}$, we want to study the entanglement under the cut $A_{i_{1}}: A_{i_{2}}: \ldots: A_{i_{l}}:\left(A_{j_{1}} \ldots A_{j_{k-l}}\right)$, where $\left\{i_{1}, \ldots, i_{l}\right\} \cup\left\{j_{1}, \ldots j_{k-l}\right\}=\{1, \ldots k\}$. We can define the set $V_{A_{i_{1}}: \ldots: A_{i_{l}}}^{k}(\rho)$ similarly.

Theorem 1. Let $T=U_{A} \otimes U_{B}$, where $U_{A}$ and $U_{B}$ are the unitary transformations on $H_{A}^{m}$ and $H_{B}^{n}$ respectively. Then $V_{A}^{k}(T(\rho))=U_{A}^{-1}\left(V_{A}^{k}(\rho)\right)$, that is $V_{A}^{k}(\rho)$ (resp. $\left.V_{B}^{k}(\rho)\right)$ is an "invariant" up to a linear (unitary if Fubuni-Study metric is used) transformation of $C P^{m-1}$ of the mixed state $\rho$ under local unitary transformations.

Proof. Let $U_{A}=\left(u_{i j}^{A}\right)_{1 \leq i \leq m, 1 \leq j \leq m}$ and $U_{B}=\left(u_{i j}^{B}\right)_{1 \leq i \leq n, 1 \leq j \leq n}$ be the matrices in the standard orthogonal basis. Then the matrix of $T(\rho)$ under the standard orthogonal base $\{|11\rangle, \ldots,|1 n\rangle, \ldots,|m 1\rangle, \ldots,|m n\rangle\}$, as a blocked matrix, is $T(\rho)=\left(\Sigma_{l, h} u_{i l}^{A} U_{B}\left(\rho_{l h}\right) U_{B}^{\dagger}\left(u_{j h}^{A}\right)^{\dagger}\right)_{1 \leq i \leq m, 1 \leq j \leq m}$. Hence

$$
V_{A}^{k}(T(\rho))=\left\{\left(r_{1}, \ldots, r_{m}\right): \operatorname{rank}\left(\Sigma_{l, h}\left(\Sigma_{i} r_{i} u_{i l}^{A}\right)\left(\Sigma_{j} r_{j} u_{j h}^{A}\right)^{\dagger} U_{B}\left(\rho_{l h}\right) U_{B}^{\dagger}\right) \leq k\right\}
$$

We set $r_{l}^{\prime}=\Sigma_{i} r_{i} u_{i l}^{A}$ for $l=1, \ldots, m$. Then 


$$
\begin{aligned}
& \Sigma_{l, h}\left(\Sigma_{i} r_{i} u_{i l}^{A}\right)\left(\Sigma_{j} r_{j} u_{j h}^{A}\right)^{\dagger} U_{B}\left(\rho_{l h}\right) U_{B}^{\dagger} \\
& \quad=U_{B}\left(\Sigma_{l h} r_{l}^{\prime}\left(r_{h}^{\prime}\right)^{\dagger}\left(\rho_{l h}\right)\right) U_{B}^{\dagger}
\end{aligned}
$$

Thus

$$
V_{A}^{k}(T(\rho))=\left\{\left(r_{1}, \ldots, r_{m}\right): \operatorname{rank}\left(\Sigma_{l h} r_{l}^{\prime}\left(r_{h}^{\prime}\right)^{\dagger}\left(\rho_{l h}\right)\right) \leq k\right\}
$$

and our conclusion follows.

Theorem 1'. Let $T=U_{A} \otimes U_{B} \otimes U_{C}$, where $U_{A}, U_{B}$ and $U_{C}$ are unitary transformations on $H_{A}^{m}, H_{B}^{n}$ and $H^{l}$ respectively. Then $V_{A: B}^{k}(T(\rho))=$ $U_{A}^{-1} \times U_{B}^{-1}\left(V_{A: B}^{k}(\rho)\right)$, that is $V_{A: B}^{k}(\rho)$ is an "invariant" up to a linear (unitary if product Fubini-Study metric is used) transformation of $C P^{m-1} \times C P^{n-1}$ of the mixed state $\rho$ under local unitary transformations.

Proof. Let $U_{A}=\left(u_{i j}^{A}\right)_{1 \leq i \leq m, 1 \leq j \leq m}, U_{B}=\left(u_{i j}^{B}\right)_{1 \leq i \leq n, 1 \leq j \leq n}$ and $U_{C}=$ $\left(u_{i j}^{C}\right)_{1 \leq i \leq l, 1 \leq j \leq l}$, be the matrix in the standard orthogonal basis.

Recall the proof of Theorem 1, we have $V_{A B}(T(\rho))=\left(U_{A} \otimes U_{B}\right)^{-1}\left(V_{A B}(\rho)\right)$ under the coordinate change

$$
\begin{gathered}
r_{k w}^{\prime}=\Sigma_{i j} r_{i j} u_{i k}^{A} u_{j w}^{B} \\
=\Sigma_{i j} r_{i}^{1} r_{j}^{2} u_{i k}^{A} u_{j w}^{B} \\
=\Sigma_{i j}\left(r_{i}^{1} u_{i k}^{A}\right)\left(r_{j}^{2} u_{j w}^{B}\right) \\
=\left(\Sigma_{i} r_{i}^{1} u_{i k}^{A}\right)\left(\Sigma_{j} r_{j}^{2} u_{j w}^{B}\right)
\end{gathered}
$$

for $k=1, \ldots, m, w=1, \ldots, n$. Thus our conclusion follows from the definition.

Similarly, we have the following result in general case.

Theorem 1". Let $T=U_{A_{i_{1}}} \otimes \cdots \otimes U_{A_{i_{l}}} \otimes U_{j_{1} \ldots j_{k-l}}$, where $U_{A_{i_{1}}}, \ldots, U_{A_{i_{l}}}, U_{j_{1} \ldots j_{k-l}}$ are unitary transformations on $H_{A_{i_{1}}}^{m_{i_{1}}}, \ldots, H_{A_{i_{l}}}^{m_{i_{l}}}$ and $\left(H_{A_{j_{1}}}^{m_{j_{1}}} \otimes \ldots \otimes H_{A_{j_{k-l}}}^{m_{j_{k}-l}}\right)$ respectively. Then $V_{A_{i_{1}}: \ldots: A_{i_{l}}}^{k}(T(\rho))=U_{A_{i_{1}}}^{-1} \times \cdots \times U_{A_{i_{l}}}^{-1}\left(V_{A_{i_{1}} \ldots: \ldots A_{i_{l}}}^{k}(\rho)\right)$.

Remark 1. Since $U_{A_{i_{1}}}^{-1} \times \cdots \times U_{A_{i_{l}}}^{-1}$ certainly preserves the standard Euclid metric of complex linear space and hence the (product) Fubini-Study metric of the product of projective complex spaces, all metric properties of $V_{A_{i_{1}}: \ldots: A_{i_{l}}}^{k}(\rho)$ are preserved when the local unitary transformations are applied to the mixed state $\rho$.

Moreover from the proof it is easy to see that all algebraic-geometric properties (since $V_{A}^{k}(\rho), V_{B}^{k}(\rho)$ are algebraic sets as proved in Theorem 2) of $V_{A}^{k}(\rho)$ (resp. $\left.V_{B}^{k}(\rho)\right)$ are preserved even under local linear inversible transformations (i.e., $T=T_{A} \otimes T_{B}$ where $T_{A}, T_{B}$ are just linear inversible operators of $H_{A}^{m}, H_{B}^{n}$.) 
We observe $V_{A}^{k}\left(\rho^{P T}\right)=\left(V_{A}^{k}(\rho)\right)^{\dagger}$, here $\dagger$ is the conjugate mapping of $C P^{m-1}$ defined by $\left(r_{1}, \ldots, r_{m}\right)^{\dagger}=\left(r_{1}^{\dagger}, \ldots, r_{m}^{\dagger}\right)$. It is clear that this property holds for other $V$ 's invariants.

Theorem 2. $V_{A}^{k}(\rho)$ (resp. $V_{B}^{k}(\rho)$ ) is an algebraic set in $C P^{m-1}$ (resp. $\left.C P^{n-1}\right)$.

From Definition 2 and Theorem 2 we immediately have the following result.

Theorem 2'. $V_{A: B}^{k}(\rho)$ is an algebraic set in $C P^{m-1} \times C P^{n-1}$.

The general result can be stated as follows.

Theorem 2". $V_{A_{i_{1}}: \ldots: A_{i_{l}}}^{k}(\rho)$ is an algebraic set in $C P^{m_{i_{1}}-1} \times C P^{m_{i_{l}}-1}$.

It is easy to see from Definitions that we just need to prove Theorem 2.

Theorem 3. If $\rho$ is a separable mixed state, $V_{A}^{k}(\rho)$ (resp. $\left.V_{B}^{k}(\rho)\right)$ is a linear subset in $C P^{m-1}$ (resp. $C P^{n-1}$ ), i.e., it is the sum of the linear subspaces.

In the following statement we give the separability criterion of the mixed state $\rho$ under the cut A:B:C. The "linear subspace of $C P^{m-1} \times C P^{n-1}$ " means the product of a linear subspace in $C P^{m-1}$ and a linear subspace in $C P^{n-1}$.

Theorem 3'. If $\rho$ is a separable mixed state on $H=H_{A}^{m} \otimes H_{B}^{n} \otimes H_{C}^{l}$ under the cut $A: B: C, V_{A: B}^{k}(\rho)$ is a linear subset in $C P^{m-1} \times C P^{n-1}$, i.e., it is the sum of the linear subspaces.

The general result can be stated as follows.

Theorem 3". If $\rho$ is a separable mixed state on $H=H_{A_{1}}^{m_{1}} \otimes \cdots \otimes H_{A_{k}}^{m_{k}}$ under the cut $A_{i_{1}}: A_{i_{2}}: \ldots: A_{i_{l}}:\left(A_{j_{1}} \ldots A_{j_{k-l}}\right), V_{A_{i_{1}}: \ldots: A_{i_{l}}}^{k}(\rho)$ is a linear subset in $C P^{m_{i_{1}}-1} \times \cdots \times C P^{m_{i_{l}}-1}$,i.e., it is the sum of the linear subspaces.

We just prove Theorem 3 and Theorem 3'. The proof of Theorem 3" is similar.

For the purpose to prove Theorem 2 and 3 we need the following lemmas.

Lemma 1. Let $\left\{e_{1}, \ldots, e_{h}\right\}$ be the orthogonal basis of a $h$ dimension Hilbert space $H, \rho=\Sigma_{l=1}^{t} p_{l} P_{v_{l}}$, where $v_{l}$ is unit vector in $H$ for $l=1, \ldots, t, v_{l}=$ $\sum_{k=1}^{h} a_{k l} e_{k}$ and $A=\left(a_{k l}\right)_{1 \leq k \leq h, 1 \leq l \leq t}$ is the $h \times t$ matrix. Then the matrix of $\rho$ with the base $\left\{e_{1}, \ldots, e_{h}\right\}$ is $A P A^{\dagger}$, where $P$ is the diagonal matrix with diagonal entries $p_{1}, \ldots, p_{h}$. 
Proof. We note that the matrix of $P_{v_{l}}$ with the basis is $\alpha_{l} \alpha_{l}^{\dagger}$ where $\alpha_{l}=$ $\left(a_{1 l}, \ldots, a_{h l}\right)^{\tau}$ is just the expansion of $v_{l}$ with the basis. The conclusion follows immediately.

The following conclusion is a direct matrix computation from Lemma 1 or see [25].

Corollary 1. Suppose $p_{i}>0$, then the image of $\rho$ is the linear span of vectors $v_{1}, \ldots, v_{t}$.

Now let $H$ be the $H_{A}^{m} \otimes H_{B}^{n},\left\{e_{1}, \ldots, e_{m n}\right\}$ be the standard orthogonal base $\left\{|11\rangle, \ldots,\left|1 n\llcorner, \ldots,|m 1\rangle, \ldots,|m n\rangle\}\right.\right.$ and $\rho=\Sigma_{l=1}^{t} p_{l} P_{v_{l}}$ with positive $p_{l}$ 's be a mixed state on $H$. We may consider the $m n \times t$ matrix $A$ as a $m \times 1$ blocked matrix with each block $A_{w}$, where $w=1, \ldots, m$, a $n \times t$ matrix corresponding to $\{|w 1\rangle, \ldots,|w n\rangle\}$. Then it is easy to see $\rho_{i j}=A_{i} P A_{j}^{\dagger}$, where $i=1, \ldots m, j=1, \ldots, m$. Thus

$$
\Sigma r_{i} r_{j}^{\dagger} \rho_{i j}=\left(\Sigma r_{i} A_{i}\right) P\left(\Sigma r_{i} A_{i}\right)^{\dagger}
$$

Lemma 2. $\Sigma r_{i} r_{j}^{\dagger} \rho_{i j}$ is a (semi) positive definite $n \times n$ matrix. Its rank equals to the rank of $\left(\Sigma r_{i} A_{i}\right)$.

Proof. The first conclusion is clear. The matrix $\Sigma r_{i} r_{j}^{\dagger} \rho_{i j}$ is of rank $k$ if and only if there exist $n-k$ linear independent vectors $c^{j}=\left(c_{1}^{j}, \ldots, c_{n}^{j}\right)$ with the property.

$$
\begin{gathered}
c^{j}\left(\Sigma_{i j} r_{i} r_{j}^{\dagger} \rho_{i j}\right)\left(c^{j}\right)^{\dagger}= \\
\left(\Sigma_{i} r_{i} c^{j} A_{i}\right) P\left(\Sigma_{i} r_{i} c^{j} A_{i}\right)^{\dagger}=0
\end{gathered}
$$

Since $P$ is a strictly positive definite matrix, our conclusion follows immediately.

Proof of Theorem 2. From Lemma 1 , we know that $V_{A}^{k}(\rho)$ is the zero locus of all $(k+1) \times(k+1)$ submatrices of $\left(\Sigma r_{i} A_{i}\right)$. The conclusion is proved.

Because the determinants of all $(k+1) \times(k+1)$ submatrices of $\left(\Sigma r_{i} A_{i}\right)$ are homogeneous polynomials of degree $k+1$, thus $V_{A}^{k}(\rho)$ (resp. $\left.V_{B}^{k}(\rho)\right)$ is an algebraic subset (called determinantal varieties in algebraic geometry [18],[20]) in $C P^{m-1}\left(\right.$ resp. $\left.C P^{n-1}\right)$.

The point here is: for different representations of $\rho$ as $\rho=\Sigma_{j} p_{j} P_{v_{j}}$ with $p_{j}$ 's positive real numbers, the determinantal varieties from their corresponding $\Sigma_{i} r_{i} A_{i}$ 's are the same. 
Now suppose that the mixed state $\rho$ is separable, i.e there are unit product vectors $a_{1} \otimes b_{1}, \ldots, a_{s} \otimes b_{s}$ such that $\rho=\sum_{l=1}^{s} q_{l} P_{a_{l} \otimes b_{l}}$, where $q_{1}, \ldots q_{s}$ are positive real numbers. Suppose $a_{u}=a_{u}^{1}|1\rangle+\ldots+a_{u}^{m}|m\rangle, b_{u}=b_{u}^{1}|1\rangle+\ldots+b_{u}^{n}|n\rangle$ for $u=1, \ldots, s$. Hence the vector representation of $a_{u} \otimes b_{u}$ with the standard basis is $a_{u} \otimes b_{u}=\Sigma_{i j} a_{u}^{i} b_{u}^{j}|i j\rangle$. Consider the corresponding $m n \times s$ matrix $C$ of $a_{1} \otimes b_{1}, \ldots, a_{s} \otimes b_{s}$ as in Lemma 1, we have $\rho=C Q C^{\dagger}$, where $Q$ is diagonal matrix with diagonal entries $q_{1}, \ldots, q_{s}$. As before we consider $C$ as $m \times 1$ blocked matrix with blocks $C_{w}, w=1, \ldots m$. Here $C_{w}$ is a $n \times s$ matrix of the form $C_{w}=\left(a_{j}^{w} b_{j}^{i}\right)_{1 \leq i \leq n, 1 \leq j \leq s}=B T_{w}$, where $B=\left(b_{j}^{i}\right)_{1 \leq i \leq n, 1 \leq j \leq s}$ is a $n \times s$ matrix and $T_{w}$ is a diagonal matrix with diagonal entries $a_{1}^{w}, \ldots, a_{s}^{w}$. Thus from Lemma 1, we have $\rho_{i j}=C_{i} Q C_{j}^{\dagger}=B\left(T_{i} Q T_{j}^{\dagger}\right) B^{\dagger}=B T_{i j} B^{\dagger}$, where $T_{i j}$ is a diagonal matrix with diagonal entries $q_{1} a_{1}^{i}\left(a_{1}^{j}\right)^{\dagger}, \ldots, q_{s} a_{s}^{i}\left(a_{s}^{j}\right)^{\dagger}$.

Proof of Theorem 3. As in the proof of Theorem 2, we have

$$
\begin{aligned}
& \Sigma r_{i} r_{j}^{\dagger} \rho_{i j}=\Sigma r_{i} r_{j}^{\dagger} B T_{i j} B^{\dagger} \\
& \quad=B\left(\Sigma r_{i} r_{j}^{\dagger} T_{i j}\right) B^{\dagger}
\end{aligned}
$$

Here we note $\Sigma r_{i} r_{j}^{\dagger} T_{i j}$ is a diagonal matrix with diagonal entries $q_{1}\left(\Sigma r_{i} a_{1}^{i}\right)\left(\Sigma r_{i} a_{1}^{i}\right)^{\dagger}, \ldots, q_{s}\left(\Sigma r_{i} a_{s}^{i}\right)\left(\Sigma r_{i} a_{s}^{i}\right)^{\dagger}$. Thus $\Sigma r_{i} r_{j}^{*} \rho_{i j}=B G Q G^{\dagger} B^{\dagger}$, where $G$ is a diagonal matrix with diagonal entries $\Sigma r_{i} a_{1}^{i}, \ldots, \Sigma r_{i} a_{s}^{i}$. Because $Q$ is a strictly positive definite matrix, from Lemma 1 we know that $\Sigma r_{i} r_{j}^{\dagger} \rho_{i j}$ is of rank smaller than $k+1$ if and only if the rank of $B G$ is strictly smaller than $k+1$. Note that $B G$ is just the multiplication of $s$ diagonal entries of $G$ (which are linear forms of $\left.r_{1}, \ldots, r_{m}\right)$ on the $s$ columns of $B$, thus the determinants of all $(k+1) \times(k+1)$ submatrices of $B G$ (in the case $s \geq k+1$, otherwise automatically linear)are the multiplications of a constant (possibly zero) and $k+1$ linear forms of $r_{1}, \ldots, r_{m}$. Thus the conclusion is proved.

From Lemma 1 and the proof of Theorem 2 and $3, \Sigma_{i} r_{i} A_{i}$ play a key role. If we take the standard $\rho=\Sigma_{j=1}^{r} p_{j} P_{\varphi_{j}}$, where $p_{j}, \varphi_{j}, j=1, \ldots, r$ are eigenvalues and eigenvectors, the corresponding $\Sigma_{i} r_{i} A_{i}$ measures the geometric positions of eigenvectors in $H_{A}^{m} \otimes H_{B}^{n}$. It is obvious from the proof of Theorem 2, the nonlocal invariants defined in Definition 1 are independent of $p_{1}, \ldots, p_{r}$, the global eigenvalue spectra of the mixed states.

Proof of Theorem 3'. We first consider the separability of $\rho$ under the cut AB:C, i.e., $\rho=\Sigma_{f=1}^{g} p_{f} P_{a_{f} \otimes b_{f}}$, where $a_{f} \in H_{A}^{m} \otimes H_{B}^{n}$ and $b_{f} \in H_{C}^{l}$ for $f=1, \ldots, g$. Consider the separability of $\rho$ under the cut A:B:C, we have $a_{f}=a_{f}^{\prime} \otimes a_{f}^{\prime \prime}, a_{f}^{\prime} \in C_{A}^{m}, a_{f}^{\prime \prime} \in C_{B}^{m}$. Let $a_{f}=\left(a_{f}^{1}, \ldots, a_{f}^{m n}\right), a_{f}^{\prime}=\left(a_{f}^{\prime 1}, \ldots, a_{f}^{\prime m}\right)$ and $a_{f}^{\prime \prime}=\left(a_{f}^{\prime \prime 1}, \ldots, a_{f}^{\prime \prime n}\right)$ be the coordinate forms with the standard orthogonal basis $\{|i j\rangle\},\{|i\rangle\}$ and $\{|j\rangle\}$ respectively, we have that $a_{f}^{i j}=a_{f}^{\prime i} a_{f}^{\prime \prime j}$. Recall the proof of Theorem 3, the diagonal entries of $G$ in the proof of Theorem 3 are 


$$
\begin{gathered}
\sum_{i j} r_{i j} a_{f}^{i j}= \\
\Sigma_{i j} r_{i}^{1} a_{f}^{\prime i} r_{j}^{2} a_{f}^{\prime \prime j}= \\
\left(\Sigma_{i} r_{i}^{1} a_{f}^{\prime i}\right)\left(\Sigma_{j} r_{j}^{1} a_{f}^{\prime \prime j}\right)
\end{gathered}
$$

Thus as argued in the proof of Theorem $3, V_{A: B}^{k}(\rho)$ has to be the zero locus of the multiplications of the linear forms in (13). The conclusion is proved.

\section{Numerical invariants}

It is a standard fact in algebraic geometry that $V^{\prime} s$ defined in section 2 are the sum of irreducible algebraic varieties( components). Suppose $V_{A}^{k}(\rho)=$ $V_{1} \cup \cdots \cup V_{t}$. From Theorem 1 and Remark 1, we know that $t$ is a numerical invariant of $\rho$ when local linear inversible transformations are applied to $\rho$. Actually, since there are a lot of numerical algebriac-geometric invariants of these components, e.g., dimensions, cohomology classes (represented by $V_{i}$ 's in $H^{*}\left(C P^{m-1}\right)$, cohomology rings of $V_{i}$ 's, etc. We can get many numerical invarints of the mixed state when local linear inversible transformations are applied to them. In this way, we get a very powerful tool of numerical invarints to distinguish the entangled classes of the mixed states in a composite quantum systems.

On the other hand, if local unitary transformations are applied to the mixed states, it is known that even the metric properties of $V^{\prime} s$ (the metric on $V$ is from the standard Fubini-Study metric of projective spaces) are invariant. Thus any complex differential geometric quantity, such as, the volumes of $V_{i}$ 's, the integrations (over the whole component) of some curvature functions of $V_{i}$ 's, are the invariants of the mixed states under local unitary transformations.

For any given pure state $|v\rangle$ in a bipartite quantum system, $|v\rangle \in H_{A}^{m} \otimes$ $H_{B}^{n}$, there exist orthogonal basis $\left|\phi_{1}\right\rangle, \ldots,\left|\phi_{m}\right\rangle$ of $H_{A}^{m}$ and orthogonal basis $\left|\psi_{1}\right\rangle, \ldots,\left|\psi_{n}\right\rangle$ of $H_{B}^{n}$, such that, $|v\rangle=\lambda_{1}\left|\phi_{1}\right\rangle \otimes\left|\psi_{1}\right\rangle+\cdots+\lambda_{d}\left|\phi_{d}\right\rangle \otimes\left|\psi_{d}\right\rangle$, where $d \leq \min \{m, n\}$. This is Schmidt decomposition (see [39]). It is clear that $d$ is an invariant under local unitary transformations. This number is called the Schmidt rank of the pure state $|v\rangle$. It is clear that $|v\rangle$ is separable if and only if its Schmidt rank is 1. Schmidt rank of pure states in a bipartite quantum systems is a classical concept in the theory of quantum entanglement, it is actually the codimension of the invariant $V_{A}^{0}(\rho)$ for the pure state $\rho=P_{|v\rangle}$.

Let $\rho=P_{|v\rangle}$ be a pure state in $H_{A}^{m} \otimes H_{B}^{n}$ with $m \leq n$. From Theorem 1 about the invariance of $V_{A}^{0}(\rho)$, we can compute it from its Schmidt decomposition $|v\rangle=\Sigma_{i=1}^{d} \lambda_{i}\left|\phi_{i}\right\rangle \otimes\left|\psi_{i}\right\rangle$. It is clear that $V_{A}^{0}(\rho)=\left\{\left(r_{1}, \ldots, r_{m}\right) \in C P^{m-1}\right.$ : $\left.\left(\lambda_{1} r_{1}, \ldots, \lambda_{d} r_{d}, 0, \ldots, 0\right)^{\tau}=0\right\}$. 
Proposition 1. For the pure state $\rho=P_{|v\rangle}, d=m$ if and only if $V_{A}^{0}(\rho)=\emptyset$ and $d=m-1-\operatorname{dim}\left(V_{A}^{0}(\rho)\right)=n-1-\operatorname{dim}\left(V_{B}^{0}(\rho)\right)$ if $d \leq m-1$.

In this way we show that the Schmidt rank of a pure state is just the codimension of the algebraic set, and thus it seems interesting to study the quantity $m-1-\operatorname{dim}\left(V_{A}^{k}(\rho)\right)$ for mixed states, since it is non-local invariant and the generalization of the classical concept of Schmid rank of pure states.

In [41] the concept of Schmidt numbers of mixed states was introduced as the minimum Schmidt rank of pure states that are needed to construct such mixed states. For a bipartite mixed state $\rho$, it has Schmidt number $k$ if and only if for any decomposition $\rho=\Sigma_{i} p_{i} P_{v_{i}}$ with positive real numbers $p_{i}$ 's and pure states $v_{i}$ 's, at least one of the pure states $v_{i}$ 's has Schmidt rank at least $k$, and there exists such a decomposition with all pure states $v_{i}$ 's Schmidt rank at most $k$. It is clear that $\rho$ is separable if and only if its Schmidt number is 1 .

From our invariants, a LOWER bound of Schmidt numbers of mixed states is given as in the following Theorem 4, which implies that the generic rank $m$ mixed states on $H_{A}^{m} \otimes H_{B}^{m}$ have relatively high Schmidt numbers and thus entangled. This is an extension of our previous results in [14].

Theorem 4. Let $\rho$ be a mixed state on $H_{A}^{m} \otimes H_{B}^{m}$ of rank $r$ and Schmidt number $k$. Suppose $V_{A}^{m-t}(\rho)=\emptyset$, then $k \geq \frac{m}{r-m+t}$.

Proof. Take any representation $\rho=\Sigma_{i=1}^{t} p_{i} P_{v_{i}}$ with $p_{i}$ 's positive, and the maximal Schmidt rank of $v_{i}$ 's is $k$. We observe that it is only needed to take $r$ linear independent vectors in $\left\{v_{1}, \ldots, v_{t}\right\}$ to compute the rank of $\Sigma_{i} r_{i} A_{i}$ in Lemma 1 , since the columns in $\Sigma_{i} r_{i} A_{i}$ corresponding to the remaining $t-r$ vectors are linear dependent on the columns corresponding to these $r$ linear independent vectors. For the purpose that the rank of these $r$ columns in $\Sigma_{i} r_{i} A_{i}$ is not bigger than $m-t$, we just need $r-m+t$ of these columns to be zero. On the other hand, from Proposition 1, the dimension of the linear subspace $\left(r_{1}, \ldots, r_{m}\right) \in H_{A}^{m}$, such that the corresponding column of $v_{i}$ in $\Sigma_{i} r_{i} A_{i}$ is zero, is exactly $m-k\left(v_{i}\right)$, where $k\left(v_{i}\right)$ is the Schmidt rank of $v_{i}$. Thus we know that there is at least one nonzero $\left(r_{1}, \ldots, r_{m}\right)$ such that $\Sigma_{i} r_{i} A_{i}$ is of rank smaller than $m-t+1$ if $m>k(r-m+t)$. The conclusion is proved.

The physical implication of Theorem 4 is interesting. We apply it to the rank $l t$ mixed states on $H_{A}^{l t} \otimes H_{B}^{l t}$ with $t \geq l$. From the Proposition in p.67 of [4] (or see Proposition 4 below), $V_{A}^{(l-1) t}(\rho)$ of the "generic" rank $l t$ mixed states $\rho$ 's has codimension $t^{2}>l t-1$ in $C P^{l t-1}$, thus empty. We know that the Schmidt numbers of these generic rank $l t$ mixed states are at least $l$. Hence we have the following result.

Corollary 2. For rank $n^{2}$ generic mixed states in a bipartite quantum sys- 
tem $H_{A}^{n^{2}} \otimes H_{B}^{n^{2}}$, their Schmidt numbers are greater than or equal to $n$.

For example, to construct "generic" rank 9 mixed states on $H_{A}^{9} \otimes H_{B}^{9}$, pure states of Schmidt rank at least 3 have to be used.

For any pure state in a bipartite quantum system $H=H_{A}^{m} \otimes H_{B}^{n}$, it can be written as a linear combination of at most $\min \{m, n\} 2$-way orthogonal separable pure states $([39],[8])$ from Schmidt decomposition. For multipartite pure states, there is no direct generaliztion of Schmidt decomposition, and those multipartite pure states with m-way orthogonal decompositions can be distilled to cat states (see [8]). From the results in [2], it is known that we need at least 5 terms of "orthogonal" separable pure states to write a generic pure state in $H_{A}^{2} \otimes H_{B}^{2} \otimes H_{C}^{2}$ as a linear combination of them. This phenomenon is a remarkable difference between bipartite pure state entanglement and multipartite pure state entanglement. In the following statement it is showed what happens for generic pure states in $H_{A}^{n^{2}} \otimes H_{B}^{n^{2}} \otimes H_{C}^{n^{2}}$.

Theorem 5. For a generic pure state $|\psi\rangle=\Sigma_{i=1}^{n^{2}} \lambda_{i}\left|\psi_{i}^{12}\right\rangle \otimes\left|\psi_{i}^{3}\right\rangle$ in a tripartite quantum system $H_{A}^{n^{2}} \otimes H_{B}^{n^{2}} \otimes H_{C}^{n^{2}}$, where $\left|\psi_{1}^{3}\right\rangle, \ldots,\left|\psi_{n^{2}}^{3}\right\rangle$ are mutually orthogonal unit vectors in $H_{C}^{n^{2}}$ and $\left|\psi_{1}^{12}\right\rangle, \ldots,\left|\psi_{n^{2}}^{12}\right\rangle$ are pure states in $H_{A}^{n^{2}} \otimes H_{B}^{n^{2}}$, then there exists one of $\left|\psi_{1}^{12}\right\rangle, \ldots,\left|\psi_{n^{2}}^{12}\right\rangle$ with Schmidt rank at least $n$.

Proof. It is clear that $\operatorname{tr}_{C}\left(P_{|\psi\rangle}\right)=\Sigma_{i=1}^{n^{2}}\left|\lambda_{i}\right|^{2} P_{\left|\psi_{i}^{12}\right\rangle}$ is a generic rank $n^{2}$ mixed state in $H_{A}^{n^{2}} \otimes H_{B}^{n^{2}}$. From Theorem 4 , at least one of $\left|\psi_{1}^{12}\right\rangle, \ldots,\left|\psi_{n^{2}}^{12}\right\rangle$ has Schmidt rank (as a pure state in $H_{A}^{n^{2}} \otimes H_{B}^{n^{2}}$ ) at least $n$. Thus our conclusion is proved.

\section{Examples}

Now we give some examples to show how to use Theorem 1,2,3 to construct and distinguish the entangled classes of the mixed states.

Example 3. Let $H=H_{A}^{m} \otimes H_{B}^{n}$ and $\rho_{a_{1}, \ldots, a_{n}}=\frac{1}{n}\left(\sum_{i=1}^{n} P_{a_{i} \otimes|i\rangle}\right)$, where $a_{i}$, $i=1, \ldots, n$, are unit vectors in $H_{A}^{m}$. This is a rank $\mathrm{n}$ separable mixed state. Suppose $a_{i}=\left(a_{i}^{1}, \ldots, a_{i}^{m}\right), i=1, \ldots, n$, the expansion with respect to the standard basis $|1\rangle, \ldots,|m\rangle$ of $H_{A}^{m}$. Let $l_{i}\left(r_{1}, \ldots, r_{m}\right)=a_{i}^{1} r_{1}+\ldots+a_{i}^{m} r_{m}$ for $i=1, \ldots, n$ be $\mathrm{n}$ linear forms. It is easy to check that $V_{A}^{n-1}(\rho)=\left\{\left(r_{1}, \ldots, r_{m}\right): l_{1} \cdots l_{n}=0\right\}$.

Proposition 2. The mixed states $\rho_{a_{1}, \ldots, a_{n}}$ and $\rho_{b_{1}, \ldots, b_{n}}$ are equivalent under the local unitary transformations if and only if there exists a unitary transformation $U_{A}$ on $H_{A}^{m}$ such that the $n$ vectors $b_{1}, . ., b_{n}$ are exactly $U_{A}\left(a_{1}\right), \ldots, U_{A}\left(a_{n}\right)$, i.e., $b_{i}=U_{A}\left(a_{i_{j}}\right)$, where $\left\{i_{1}, \ldots, i_{n}\right\}=\{1, \ldots, n\}$. 
Proof. The "if" part is clear. Let $l_{i}^{\prime}\left(r_{1}, \ldots, r_{m}\right)=b_{i}^{1} r_{1}+\ldots+b_{i}^{m} r_{m}$ for $i=1, \ldots, n$. Then $V_{A}^{n-1}\left(\rho_{a_{1}, \ldots, a_{n}}\right)$ (resp. $V_{A}^{n-1}\left(\rho_{b_{1}, \ldots, b_{n}}\right)$ )are the union of $\mathrm{n}$ hyperplanes defined by $l_{i}=0$ (resp. $\left.l_{i}^{\prime}=0\right)$ for $i=1, \ldots, n$. It should be noted here these hyperplances are counted by multiplicities. From Theorem 1 we get the conclusion.

Segre variety $\Sigma_{n, m}$, which is the image of the following map, $\sigma: C P^{n} \times$ $C P^{m} \rightarrow C P^{(n+1)(m+1)-1}$ where $\sigma\left(\left[X_{0}, \ldots, X_{n}\right],\left[Y_{0}, \ldots, Y_{m}\right]\right)=\left[\ldots, X_{i} Y_{j}, \ldots\right]$, is a famous determinantal variety (see [23], pp.25-26). It is clear that Segre variety is irreducible and not a linear subvariety. We consider the Segre variety $\Sigma_{1, m}$ in the case $n=1$, actually $\Sigma_{1, m}=\left\{\left(r_{1}, \ldots, r_{2 n}\right): \operatorname{rank}(M) \leq 1\right\}$ where $M$ is the following matrix

$$
\left(\begin{array}{cccc}
r_{1} & r_{2} & \ldots & r_{n} \\
r_{n+1} & r_{n+2} & \ldots & r_{2 n}
\end{array}\right)
$$

Example 4 (entangled mixed state from Segre variety). Let $H=$ $H_{A}^{2 m} \otimes H_{B}^{2},\left|\phi_{i}\right\rangle=\frac{1}{\sqrt{2}}(|i 1\rangle+|(m+i) 2\rangle)$ for $i=1, \ldots, m$ and $\rho=\frac{1}{m}\left(P_{\left|\phi_{1}\right\rangle}+\ldots+\right.$ $\left.P_{\left|\phi_{m}\right\rangle}\right)$. This is a mixed state of rank $m$. By computing $\Sigma_{i} r_{i} A_{i}$ as in the proof of Theorem 2, we get $V_{A}^{1}(\rho)=\Sigma_{1, m}$. Thus $\rho$ is an entangled mixed state.

The rank 1 locus $X_{l, n-l-1}=\{\operatorname{rank}(R) \leq 1\}$ of the following $2 \times(n-1)$ matrix $R$

$$
\left(\begin{array}{cccccc}
r_{0} & \ldots & r_{l-1} & r_{l+1} & \ldots & r_{n-1} \\
r_{1} & \ldots & r_{l} & r_{l+2} & \ldots & r_{n}
\end{array}\right)
$$

is the rational normal scroll (see p.106 [23]). The mixed states corresponding to them are as follows.

Example 5 (entangled mixed state from rational normal scroll). Let $H=H_{A}^{n+1} \otimes H_{B}^{2}$ and $\left|\phi_{1}\right\rangle=\frac{1}{\sqrt{2}}(|01>+| 12>), . .,\left|\phi_{i}\right\rangle=\frac{1}{\sqrt{2}}(\mid(i-1) 1>$ $+\mid i 2>), \ldots,\left|\phi_{l}\right\rangle=\frac{1}{\sqrt{2}}(|(l-1) 1>+| l 2>),\left|\phi_{l+1}\right\rangle=\frac{1}{\sqrt{2}}(|(l+1) 1>+|(l+2) 2>)$, $\ldots,\left|\phi_{j}\right\rangle=\frac{1}{\sqrt{2}}(|j 1>+|(j+1) 2>), \ldots,\left|\phi_{n-1}\right\rangle=\frac{1}{\sqrt{2}}(|(n-1) 1>+| n 2>)$. We consider the mixed state $\rho_{l}=\frac{1}{n-1}\left(P_{\left|\phi_{1}\right\rangle}+\ldots+P_{\left|\phi_{n-1}\right\rangle}\right)$ of rank $n-1$ on $H$. It is clear from section $2 V_{A}^{1}(\rho)=X_{l, n-l-1} \subset C P^{n}$. From the well-known fact in algebraic geometry (see pp.92-93 of [23]) we have the following result.

Proposition 3. The mixed states $\rho_{l}, l=1, \ldots,\left[\frac{n-1}{2}\right]$ are entangled and $\rho_{l}$ and $\rho_{l^{\prime}}$ for $l \neq l^{\prime}$ are not equivalent under local unitary transformations.

We need to recall a well-known result in the theory of determinantal varieties (see Proposition in p.67 of [4]). Let $M(m, n)=\left\{\left(x_{i j}\right): 1 \leq i \leq m, 1 \leq j \leq n\right\}$ (isomorphic to $C P^{m n-1}$ ) be the projective space of all $m \times n$ matrices. For a 
integer $0 \leq k \leq \min \{m, n\}, M(m, n)_{k}$ is defined as the locus $\left\{A=\left(x_{i j}\right) \in\right.$ $M(m, n): \operatorname{rank}(A) \leq k\} . M(m, n)_{k}$ is called generic determinantal varieties.

Proposition 4. $M(m, n)_{k}$ is an irreducible algebriac subvariety of $M$ of codimension $(m-k)(n-k)$.

Suppose $m \leq n$, we now construct a mixed state $\rho$ with $V_{A}^{m-1}(\rho)=M(m, n)_{(m-1)}$.

Example 6 (generic entangled mixed state). Let $H=H_{A}^{m n} \otimes H_{B}^{m}$, where $m \leq n$, and $A_{i j}, i=1, \ldots, m, j=1, \ldots n$ be $m \times n$ matrix with only nonzero entry at $i j$ position equal to 1 . Let $A$ be a blocked $m n \times 1$ matrix with $i j$ block $A_{i j}$. Here the $k$-th row of $A_{i j}$ in $A$ corresponds to the vector $|(i j) k\rangle$ in the standard basis of $H$. Hence $A$ is a size $m^{2} n \times n$ matrix. Let $\rho=\frac{1}{D} A A^{\dagger}$ be a mixed state on $H$ (Here $D$ is a normalizing constant). It is a rank $n$ mixed state.

It is easy to compute $\Sigma_{i j} r_{i j} A_{i j}=\left(r_{i j}\right)_{1 \leq i \leq m, 1 \leq j \leq n}$ (up to a constant). Thus we have $V_{A}^{m-1}(\rho)=M(m, n)_{(m-1)}$. From Proposition 4 and Theorem 3, $\rho$ is an entangled mixed state.

Example 7. Let $H=H_{A}^{m} \otimes H_{B}^{n} \otimes H_{C}^{m},\left|\phi_{l}\right\rangle=\frac{1}{m} \sum_{i=1}^{m}|i l i\rangle$ for $l=1, \ldots, n$ and $\rho=\frac{1}{n}\left(P_{\left|\phi_{1}\right\rangle}+\ldots+P_{\left|\phi_{n}\right\rangle}\right)$ be a rank $\mathrm{n}$ mixed state on $H$. It is clear that under the cut $\mathrm{B}: \mathrm{AC}, \rho$ is separable. However, under the cut AB:C, we can check that $\rho$ is just the mixed state in Example 6 and thus entangled. Similarly under the cut A:BC, $\rho$ is also the mixed state in Example 6 and thus entangled. Hence this is a mixed state on tripartite quantum system with the property that it is separable under B:AC cut and entangled under $\mathrm{AB}: \mathrm{C}$ and $\mathrm{A}: \mathrm{BC}$ cuts.

Example 8 (entangled mixed states from Eisenbud Theorem [20]). Let $H=H_{A}^{h} \otimes H_{B}^{m}$, where $h \geq n m-m+1$ and $n \geq m$ are positive integers, $A_{i}, i=1, \ldots, h$ be $m \times n$ matrix with the property: the space $M$ of linear forms (of $\left.r_{1}, \ldots, r_{h}\right)$ span by the entries of $T\left(r_{1}, \ldots, r_{h}\right)=\Sigma_{i} r_{i} A_{i}$ is of dimension $h$. Let $A$ be the $h m \times n$ matrix with $i$-th block $A_{i}$. Here the $k$-th row of $A_{i}$ in $A$ corresponds to the vector $|i k\rangle$ in the standard basis of $H$. Let $\rho=\frac{1}{D} A A^{\dagger}$ ( $D$ is a normalizing constant) be a (rank n) mixed state on $H$. From the proof of Theorem 2 we have $V_{A}^{m-1}(\rho)=\left\{\left(r_{1}, \ldots, r_{h}\right): \operatorname{rank}\left(T\left(r_{1}, \ldots, r_{h}\right)\right) \leq m-1\right\}$

Theorem 6. $\rho$ is an entangled mixed state.

Proof. It is clear that $M(m, n)$ is 1-generic (see [20], p.548) We can see that the space $M$ has codimension (in $M(m, n)$ ) smaller or equal to $m-1$ (here $v=n, w=m, k=m-1$ as refered to Theorem 2.1 in p.552 of [20]). From definition it is clear that $V_{A}(\rho)$ is just $M_{m-1}$, which is reduced and irreducible and of codimension $m-1$ in $M(m, n)$ from Theorem 2.1 of [20]. The conclusion is proved.

Eisenbud Theorem (Theorem 2.1 in [20] and thus Theorem 6 here) gives us 
a general method to construct many entangled states of low ranks, since the condition about $M$ is not a very strong restriction.

Example 9 (Bennett-DiVincenzo-Mor-Shor-Smolin-Terhal 's mixed state from UPB [7] ) Let $H=H_{A}^{2} \otimes H_{B}^{2} \otimes H_{C}^{2},\left|\phi_{+}\right\rangle=\frac{1}{\sqrt{2}}(|1\rangle+|2\rangle),\left|\phi_{-}\right\rangle=$ $\frac{1}{\sqrt{2}}(|1\rangle-|2\rangle)$. Consider the linear subspace $T$ spaned by the following 4 vectors $|1\rangle \otimes|2\rangle \otimes\left|\phi_{+}\right\rangle,|2\rangle \otimes\left|\phi_{+}\right\rangle \otimes|1\rangle,\left|\phi_{+}\right\rangle \otimes|1\rangle \otimes|2\rangle,\left|\phi_{-}\right\rangle \otimes\left|\phi_{-}\right\rangle \otimes\left|\phi_{-}\right\rangle$. Now $P$ is the projection to the complementary space $T^{\perp}$ of $T$ and $\rho=\frac{1}{D} P$ is a rank 4 PPT mixed state on $H$. It is proved in [7] that $\rho$ is entangled under the cut A:B:C (thus bound entanglement), however, it is separable under the cuts A:BC,B:AC,C:AB. Now we can compute its invariants $V_{A B}^{1}(\rho)$ and $V_{A: B}^{1}(\rho)$. It is easy to see from Theorem 3 that $V_{A B}^{1}(\rho)$ should be linear, however we can see that $V_{A: B}^{1}(\rho)$ is also linear from our computation below, though it is entangled under the cut A:B:C.

It is easy to check that the following 4 vectors $|010\rangle-|011\rangle,|100\rangle-|110\rangle$, $|001\rangle-|101\rangle,|000\rangle-|111\rangle$ are the base of $T^{\perp}$. Thus the matrix $A$ is of the following form (with rows correspond to $|000\rangle,|001\rangle,|010\rangle,|011\rangle,|100\rangle,|101\rangle,|110\rangle,|111\rangle$ )

$$
\left(\begin{array}{cccc}
0 & 0 & 0 & 1 \\
0 & 0 & 1 & 0 \\
1 & 0 & 0 & 0 \\
-1 & 0 & 0 & 0 \\
0 & 1 & 0 & 0 \\
0 & 0 & -1 & 0 \\
0 & -1 & 0 & 0 \\
0 & 0 & 0 & -1
\end{array}\right)
$$

and $\Sigma_{i j} r_{i j} A_{i j}$ is of the following form

$$
\left(\begin{array}{cccc}
r_{01} & r_{10}-r_{11} & 0 & r_{00} \\
-r_{01} & 0 & r_{00}-r_{10} & -r_{11}
\end{array}\right)
$$

Thus $V_{A B}^{1}(\rho)$ is the union of the following 3 points $(1: 1: 0: 0),(0: 1: 0$ : $1),(0: 0: 1: 0)$ in $C P^{3}$ and $V_{A: B}^{1}(\rho)$ is union of $C P^{1} \times(1: 0),(0: 1) \times C P^{1}$ and $(1: 0) \times(0: 1)$ in $C P^{1} \times C P^{1}$.

\section{Generalized Smolin state}

J.A.Smolin [40] introduced a rank 4 mixed state $\rho=\frac{1}{4}\left(P_{\left|v_{1}\right\rangle}+P_{\left|v_{2}\right\rangle}+P_{\left|v_{3}\right\rangle}+P_{\left|v_{4}\right\rangle}\right)$ on 4-party quantum system $H_{A}^{2} \otimes H_{B}^{2} \otimes H_{C}^{3} \otimes H_{D}^{2}$, where, 


$$
\begin{aligned}
& \left|v_{1}\right\rangle=\frac{1}{2}(|0000\rangle+|0011\rangle+|1100\rangle+|1111\rangle) \\
& \left|v_{2}\right\rangle=\frac{1}{2}(|0000\rangle-|0011\rangle-|1100\rangle+|1111\rangle) \\
& \left|v_{3}\right\rangle=\frac{1}{2}(|0101\rangle+|0110\rangle+|1001\rangle+|1010\rangle) \\
& \left|v_{2}\right\rangle=\frac{1}{2}(|0101\rangle-|0110\rangle-|1001\rangle+|1010\rangle)
\end{aligned}
$$

This mixed state $\rho$ is a bound entangled state when 4 parties A,B,C,D are isolated.

The following example, which is a continuous family (depending on 4 parameters) of mixed state in the 4-party quantum system $H_{A}^{2} \otimes H_{B}^{2} \otimes H_{C}^{2} \otimes H_{D}^{2}$ separable for any $2: 2$ cut but entangled for any $1: 3$ cut (thus bound entangled mixed state when A,B,C,D are isolated), can be thought as a generalization of Smolin's mixed state in [40]. We prove that the generic members in this family of mixed states are not equivalent under local unitary transformations (Theorem 7 below).

Example 10. Let $H=H_{A}^{2} \otimes H_{B}^{2} \otimes H_{C}^{2} \otimes H_{D}^{2}$ and $h_{1}, h_{2}, h_{3}, h_{4}$ (understood as row vectors) be 4 mutually orthogonal unit vectors in $C^{4}$ and $a_{1}, a_{2}, a_{3}, a_{4}$ be 4 parameters. Consider the $16 \times 4$ matrix $T$ with 16 rows as $T=\left(a_{1} h_{1}^{\tau}, 0,0, a_{2} h_{2}^{\tau}, 0, a_{3} h_{3}^{\tau}, a_{4} h_{4}^{\tau}, 0,0, a_{5} h_{4}^{\tau}, a_{6} h_{3}^{\tau}, 0, a_{7} h_{2}^{\tau}, 0,0, a_{8} h_{1}^{\tau}\right)^{\tau}$. Let $\left|\phi_{1}^{\prime}\right\rangle$, $\left|\phi_{2}^{\prime}\right\rangle,\left|\phi_{3}^{\prime}\right\rangle,\left|\phi_{4}^{\prime}\right\rangle$ be 4 vectors in $H$ whose expansions with the basis $|0000\rangle,|0001\rangle$, $|0010\rangle,|0011\rangle,|0100\rangle,|0101\rangle,|0110\rangle,|0111\rangle,|1000\rangle,|1001\rangle,|1010\rangle,|1011\rangle,|1100\rangle$, $|1101\rangle,|1110\rangle,|1111\rangle$ are exactly the 4 columns of the matrix $T$ and $\left|\phi_{1}\right\rangle,\left|\phi_{2}\right\rangle,\left|\phi_{3}\right\rangle$, $\left|\phi_{4}\right\rangle$ be the normalized unit vectors of $\left|\phi_{1}^{\prime}\right\rangle,\left|\phi_{2}^{\prime}\right\rangle,\left|\phi_{3}^{\prime}\right\rangle,\left|\phi_{4}^{\prime}\right\rangle$. Let $\rho=\frac{1}{4}\left(P_{\left|\phi_{1}\right\rangle}+\right.$ $\left.P_{\left|\phi_{2}\right\rangle}+P_{\left|\phi_{3}\right\rangle}+P_{\left|\phi_{4}\right\rangle}\right)$.

It is easy to check that when $h_{1}=(1,1,0,0), h_{2}=(1,-1,0,0), h_{3}=(0,0,1,1)$, $h_{4}=(0,0,1,-1)$ and $a_{1}=a_{2}=a_{3}=a_{4}=1$. It is just the Smolin's mixed state in $[40]$.

Now we prove that $\rho$ is invariant under the partial transposes of the cuts $\mathrm{AB}: \mathrm{CD}, \mathrm{AC}: \mathrm{BD}, \mathrm{AD}: \mathrm{BC}$.

Let the "representation" matrix $T=\left(b_{i j k l}^{t}\right)_{i=0,1, j=0,1, k=0,1, l=0,1, t=1,2,3,4}$ be the matrix with columns corresponding the expansions of $\phi_{1}, \phi_{2}, \phi_{3}, \phi_{4}$. Then we can consider that $T=\left(T_{1}, T_{2}, T_{3}, T_{4}\right)^{\tau}$ as a blocked matrix of size $4 \times 1$ with each block $T_{i j}=\left(b_{k l}^{t}\right)_{k=0,1, l=0,1,=1,2,3,4}$ a $4 \times 4$ matrix, where $i j=00,01,10,11$. Because $h_{1}, h_{2}, h_{3}, h_{4}$ are mutually orthogonal unit vectors we can easily check that $T_{i j} T_{i^{\prime} j^{\prime}}^{\dagger}=T_{i^{\prime} j^{\prime}} T_{i j}^{\dagger}$ Thus it is invariant when the partial transpose of the cut AB:CD is applied.

With the same methods we can check that $\rho$ is invariant when the partial transposes of the cuts $\mathrm{AC}: \mathrm{BD}, \mathrm{AD}: \mathrm{BC}$ are applied. Hence $\rho$ is PPT under the cuts $\mathrm{AB}: \mathrm{CD}, \mathrm{AC}: \mathrm{BD}, \mathrm{AD}: \mathrm{BC}$. Thus from a result in [30] we know $\rho$ is separable under these cuts $\mathrm{AB}: \mathrm{CD}, \mathrm{AC}: \mathrm{BD}, \mathrm{AD}: \mathrm{BC}$. 
Now we want to prove $\rho$ is entangled under the cut A:BCD by computing $V_{B C D}^{1}(\rho)$. From the previous arguments, we can check that $V_{B C D}^{1}(\rho)$ is the locus of the condition: $a_{1} h_{1} r_{000}+a_{2} h_{2} r_{011}+a_{3} h_{3} r_{101}+a_{4} h_{4} r_{110}$ and $a_{7} h_{1} r_{100}+a_{8} h_{2} r_{111}+a_{5} h_{3} r_{001}+a_{6} h_{4} r_{010}$ are linear dependent. This is equivalent to the condition that the matrix (12) is of rank 1.

$$
\left(\begin{array}{cccc}
a_{7} r_{100} & a_{8} r_{111} & a_{5} r_{001} & a_{6} r_{010} \\
a_{1} r_{000} & a_{2} r_{011} & a_{3} r_{101} & a_{4} r_{110}
\end{array}\right)
$$

From [23] pp. 25-26 we can check that $V_{B C D}^{1}(\rho)$ is exactly the famous Segre variety in algebraic geometry. It is irreducible and thus cannot be linear. From Theorem $3, \rho$ is entangled under the cut A:BCD. Similarly we can prove that $\rho$ is entangled under the cuts B:ACD, C:ABD, D:ABC.

Now we compute $V_{A: B}^{3}(\rho)$. From the previous arguments and Definition 2 , it is just the locus of the condition that the vectors $h_{1}\left(a_{1} r_{0}^{1} r_{0}^{2}+a_{7} r_{1}^{1} r_{1}^{2}\right)$, $h_{3}\left(a_{3} r_{0}^{1} r_{1}^{2}+a_{5} r_{1}^{1} r_{0}^{2}\right), h_{4}\left(a_{4} r_{0}^{1} r_{1}^{2}+a_{6} r_{1}^{1} r_{0}^{2}\right), h_{2}\left(a_{2} r_{0}^{1} r_{0}^{2}+a_{8} r_{1}^{1} r_{1}^{2}\right)$ are linear dependent. Since $h_{1}, h_{2}, h_{3}, h_{4}$ are mutually orthogonal unit vectors, we have

$$
\begin{gathered}
V_{A: B}^{3}(\rho)=\left\{\left(r_{0}^{1}, r_{1}^{1}, r_{0}^{2}, r_{1}^{2}\right) \in C P^{1} \times C P^{1}:\right. \\
\left.\left(a_{1} r_{0}^{1} r_{0}^{2}+a_{7} r_{1}^{1} r_{1}^{2}\right)\left(a_{3} r_{0}^{1} r_{1}^{2}+a_{5} r_{1}^{1} r_{0}^{2}\right)\left(a_{4} r_{0}^{1} r_{1}^{2}+a_{6} r_{1}^{1} r_{0}^{2}\right)\left(a_{2} r_{0}^{1} r_{0}^{2}+a_{8} r_{1}^{1} r_{1}^{2}\right)=0\right\}
\end{gathered}
$$

From Theorem 3 we know that $\rho$ is entangled for the cut A:B:CD, A:C:BD and A:D:BC for generic parameters, since $a_{1} r_{0}^{1} r_{0}^{2}+a_{7} r_{1}^{1} r_{1}^{2}$,etc., cannot be factorized to 2 linear forms for generic $a_{1}$ and $a_{7}$. This provides another proof that the mixed state is entangled if the 4 parties are isolated.

Let $\lambda_{1}=-a_{1} / a_{7}, \lambda_{2}=-a_{3} / a_{5}, \lambda_{3}=-a_{4} / a_{6}, \lambda_{4}=-a_{2} / a_{8}$ and consider the family of the mixed states $\left\{\rho_{\lambda_{1,2,3}, 4}\right\}$, we want to prove the following statement.

Theorem 7. The generic memebers in this continuous family of mixed states are inequivalent under the local unitary transformations on $H=H_{A}^{2} \otimes$ $H_{B}^{2} \otimes H_{C}^{2} \otimes H_{D}^{2}$.

Proof. From the above computation, $V_{A: B}^{3}\left(\rho_{\lambda_{1,2,3,4}}\right)$ is the union of the following 4 algbraic varieties in $C P^{1} \times C P^{1}$.

$$
\begin{aligned}
& V_{1}=\left\{\left(r_{0}^{1}, r_{1}^{1}, r_{0}^{2}, r_{1}^{2}\right) \in C P^{1} \times C P^{1}: r_{0}^{1} r_{0}^{2}-\lambda_{1} r_{1}^{1} r_{1}^{2}=0\right\} \\
& V_{2}=\left\{\left(r_{0}^{1}, r_{1}^{1}, r_{0}^{2}, r_{1}^{2}\right) \in C P^{1} \times C P^{1}: r_{0}^{1} r_{1}^{2}-\lambda_{2} r_{1}^{1} r_{0}^{2}=0\right\} \\
& V_{3}=\left\{\left(r_{0}^{1}, r_{1}^{1}, r_{0}^{2}, r_{1}^{2}\right) \in C P^{1} \times C P^{1}: r_{0}^{1} r_{1}^{2}-\lambda_{3} r_{1}^{1} r_{0}^{2}=0\right\} \\
& V_{4}=\left\{\left(r_{0}^{1}, r_{1}^{1}, r_{0}^{2}, r_{1}^{2}\right) \in C P^{1} \times C P^{1}: r_{0}^{1} r_{0}^{2}-\lambda_{4} r_{1}^{1} r_{1}^{2}=0\right\}
\end{aligned}
$$


From Theorem 1', if $\rho_{\lambda_{1,2,3,4}}$ and $\rho_{\lambda_{1,2,3,4}^{\prime}}$ are equivalent by a local operation, there must exist 2 fractional linear transformations $T_{1}, T_{2}$ of $C P^{1}$ such that $T=T_{1} \times T_{2}$ (acting on $C P^{1} \times C P^{1}$ ) transforms the 4 varieties $V_{1}, V_{2}, V_{3}, V_{4}$ of $\rho_{\lambda_{1,2,3,4}}$ to the 4 varieties $V_{1}^{\prime}, V_{2}^{\prime}, V_{3}^{\prime}, V_{4}^{\prime}$ of $\rho_{\lambda_{1,2,3,4}^{\prime}}$, i.e., $T\left(V_{i}\right)=V_{j}^{\prime}$.

Introduce the inhomogeneous coordinates $x_{1}=r_{0}^{1} / r_{1}^{1}, x_{2}=r_{0}^{2} / r_{1}^{2}$. Let $T_{1}\left(x_{1}\right)=\left(a x_{1}+b\right) /\left(c x_{1}+d\right)$. Suppose $T\left(V_{i}\right)=V_{i}^{\prime}, i=1,2,3,4$. Then we have $a b \lambda_{1}=c d \lambda_{1}^{\prime} \lambda_{2}^{\prime}$ and $a b \lambda_{4}=c d \lambda_{3}^{\prime} \lambda_{4}^{\prime}$. Hence $\lambda_{1} \lambda_{3}^{\prime} \lambda_{4}^{\prime}=\lambda_{1}^{\prime} \lambda_{2}^{\prime} \lambda_{4}$. This means that there are some algebraic relations of parameters if the $T$ exists. Similarly we can get the same conclusion for the other possibilities $T\left(V_{i}\right)=V_{j}^{\prime}$. This implies that there are some algebraic relations of parameters $\lambda_{1,2,3,4}$ and $\lambda_{1,2,3,4}^{\prime}$ if $\rho_{\lambda_{1,2,3,4}}$ and $\rho_{\lambda_{1,2,3,4}^{\prime}}$ are equivalent by a local unitary transformations. Hence our conclusion follows immediately.

\section{Non empty theorem}

In this section, we prove that the algebraic set invariants introduced in section 2 are not empty for low rank mixed states.

Theorem 8. Let $H=H_{A}^{m} \otimes H_{B}^{n}$ be a bipartite quantum system and $\rho$ is a rank $r$ mixed state on $H$ with $r \leq m+n-2$. Then $V_{A}^{n-1}(\rho)$ and $V_{B}^{m-1}(\rho)$ are not empty.

Proof. We take "the standard representation" $\rho=\Sigma_{i=1}^{r} p_{i} P_{v_{i}}$, where $p_{1}, \ldots, p_{r}$, $, v_{1}, \ldots, v_{r}$ are eigenvalues and eigenvectors of $\rho$ and $r=\operatorname{rank}(\rho)$. Recall the proof of Theorem $2, V_{A}^{n-1}(\rho)$ is the locus of the condition that $\Sigma_{i} r_{i} A_{i}$ has its rank smaller than $n$. Thus from Proposition 4 above we know that the codimension of $V_{A}^{n-1}(\rho)$ in $C P^{m-1}$ is smaller or equal to $(n-(n-1))(r-(n-1))=r-n+1$. Hence $\operatorname{dim}\left(V_{A}(\rho)\right) \geq m-1-r+n-1 \geq 0$ and $V_{A}^{n-1}(\rho)$ is not empty. The conclusion for $V_{B}^{m-1}(\rho)$ can be proved similarly.

\section{A relation of determinants}

As indicated in section 2, we can have the following statement from Lemma 2.

Theorem 9. Let $H=H_{A}^{m} \otimes H_{B}^{n}$ be a bipartite quantum system and $\rho=\Sigma_{l=1}^{t} p_{l} P_{v_{l}}=\Sigma_{l=1}^{s} q_{l} P_{v_{l}^{\prime}}$ be a mixed state with two "representations" as convex combinations of projections with $p_{1}, \ldots, p_{t}, q_{1}, \ldots, q_{s}>0$. Let $A$ (resp. $A^{\prime}$ ) be the $m n \times t($ resp. $m n \times s)$ matrix of vectors $v_{1}, \ldots, v_{t}\left(\right.$ resp. $\left.v_{1}^{\prime}, \ldots, v_{s}^{\prime}\right)$ in the standard basis $|11\rangle, \ldots,|1 n\rangle, \ldots,|m 1\rangle, \ldots,|m n\rangle$ as in Lemma 1. We represent $A$ (resp. $\left.A^{\prime}\right)$ as $m \times 1$ blocked matrix with blocks $A_{1}, \ldots, A_{m}\left(\right.$ resp. $\left.A_{1}^{\prime}, \ldots, A_{m}^{\prime}\right)$. Then the determinantal varieties defined by the conditions that the ranks of 
$R=\Sigma_{i} r_{i} A_{i}$ and $R^{\prime}=\Sigma_{i} r_{i} A_{i}^{\prime}$ are smaller than $k, k=0,1, \ldots, n-1$, are the same.

Actually we can get more information about the determinants of $n \times n$ submatrices of $\Sigma_{i} r_{i} A_{i}$ and $\Sigma_{i} r_{i} A_{i}^{\prime}$ from the proof of Theorem 2 and 3. This relation seems to be helpful to extract information of $\rho$ 's one unknown "representation" from its another known "representation", as in the proof of Theorem 3.

Theorem 9'. Let $H, \rho, p_{1}, \ldots p_{t}, q_{1}, \ldots, q_{s}, A, A^{\prime}, R, R^{\prime}$ be as above and $R_{i_{1}, \ldots i_{n}}$ (resp. $R_{i_{1}^{\prime}, \ldots, i_{n}^{\prime}}^{\prime}$ ) be the $n \times n$ submatrix of $R$ (resp. $R^{\prime}$ ) consisting of $i_{1}<\ldots<i_{n}$ (resp. $\left.i_{1}^{\prime}<\ldots<i_{n}^{\prime}\right)$-th columns, where $i_{1}, \ldots, i_{n} \in\{1, \ldots t\}$ and $i_{1}^{\prime}, \ldots, i_{n}^{\prime} \in\{1, \ldots, s\}$ are distinct indices. Then we have

$$
\sum_{i_{1}<\ldots<i_{n}} p_{i_{1} \ldots} \ldots p_{i_{n}}\left|R_{i_{1}, \ldots, i_{n}}\right|^{2}=\Sigma_{i_{1}^{\prime}<\ldots<i_{n}^{\prime}} q_{i_{1}^{\prime} \ldots} q_{i_{n}^{\prime}}\left|R_{i_{1}^{\prime}, \ldots, i_{n}^{\prime}}^{\prime}\right|^{2}
$$

The above result follows from the following Lemma immediately, Since both sides of the equality are just $\operatorname{det}\left(\Sigma_{i j} r_{i} r_{j}^{\dagger} \rho_{i j}\right)$.

Lemma 3 (Binet-Cauchy formula). Let $B$ be a $n \times t$ matrix with $t>n$ and $B_{i_{1}, \ldots, i_{n}}$ be the $n \times n$ submatrix of $B$ consisting of $i_{1}<\ldots<i_{n}$-th columns. Then $\operatorname{det}\left(B B^{\dagger}\right)=\Sigma_{i_{1}<\ldots<i_{n}}\left|\operatorname{det} B_{i_{1}, . ., i_{n}}\right|^{2}$.

It is clear that Theorem 2 and 3 follow from Theorem 8' here immediately.

The following result was previously known in [29],[30].

Proposition 5. Let $H=H_{A}^{n} \otimes H_{B}^{n}, \rho=\frac{1}{n} \sum_{i=1}^{n} p_{i} P_{a_{i} \otimes b_{i}}$ where $a_{1}, \ldots, a_{n}$ $\left(\right.$ resp. $\left.b_{1}, \ldots, b_{n}\right)$ are linearly independent unit vectors in $H_{A}^{n}$ (resp. $\left.H_{B}^{n}\right)$. Suppose that $\rho=\frac{1}{t} \sum_{i=1}^{t} q_{i} P_{c_{i} \otimes d_{i}}$ is another representation of $\rho$ as a convex combination with $q_{i}$ 's positive, then actually we have $t=n$ and $\left\{a_{1} \otimes b_{1}, \ldots, a_{n} \otimes b_{n}\right\}=$ $\left\{c_{1} \otimes d_{1}, \ldots, c_{n} \otimes d_{n}\right\}$.

Proof. We apply Theorem 9' to the 2 "representations" here. First of all, we know that $\Sigma_{i j} r_{i} r_{j}^{\dagger} \rho_{i j}$ is (up to a nonzero constant)the square of the absolute value of a multiplication of linear forms $b_{i}\left(r_{1}, \ldots, r_{n}\right)=\Sigma_{j} b_{1}^{j} r_{j}$, where $b_{i}=\Sigma_{j} b_{i}^{j} \mid j>$ is the coordinate form of $b_{i}$ for $i=1, \ldots, n$, from one known "representation". Thus we know from Theorem 8' that there are at least $n$ vectors in $\left\{d_{1}, \ldots, d_{t}\right\}$, without loss of generality, suppose they are $d_{1}, \ldots, d_{n}$, are just $b_{1}, \ldots, b_{n}$. Using Theorem 9' for the second factor and consider the 1st,...,n-th columns of $R^{\prime}$, this implies that the multiplication of the linear forms $c_{1}\left(r_{1}, \ldots r_{n}\right), \ldots, c_{n}\left(r_{1}, \ldots, r_{n}\right)$ are just the multiplication of the linear forms $a_{1}\left(r_{1}, \ldots, r_{n}\right), \ldots, a_{n}\left(r_{1}, \ldots, r_{n}\right)$. Hence we know that the set $\left\{c_{1}, \ldots, c_{n}\right\}$ are just the set $\left\{a_{1}, \ldots, a_{n}\right\}$. 
On the other hand it is easy to see that $a_{i} \otimes b_{j}$ with $i \neq j$ is not in the linear span of $a_{1} \otimes b_{1}, \ldots, a_{n} \otimes b_{n}$, since $a_{1}, . ., a_{n}$ (resp. $b_{1}, \ldots, b_{n}$ ) are linear independent. Thus $c_{i}=a_{i}$ from Corollary 1 .

Applying Theorem 9' to other columns of $R$ and $R^{\prime}$ by a similar argument, we have $c_{j} \in\left\{a_{1}, \ldots, a_{n}\right\}$ and $d_{j} \in\left\{b_{1}, \ldots, b_{n}\right\}$. Since $a_{i} \otimes b_{j}$ with $i \neq j$ cannot be in the image of $\rho, c_{j} \otimes d_{j}$ has to be the form $a_{i_{j}} \otimes b_{i_{j}}$. The conclusion is proved.

Remark 2. If we compute $V_{A}^{n-1}(\rho)$ from the representation of $\rho$ 's standard form, i.e., linear sum of projections to its eigenvectors, it can be seen that our invariants defined in section 2 are independent of eigenvalues $\left(p_{1}, \ldots, p_{t}\right.$ in section 2). However the information of $p_{1}, \ldots, p_{t}$ or eigenvalues is certainly reflected in Theorem 9' here. Thus Theorem 9' might be more useful in determining whether a given mixed state is entangled or not, provided that we know how to extract sufficient information from Theorem 9'.

Remark 3. As showed in Example 1, our invariants might be empty set for high rank mixed states, however it seems that Theorem 9' is still useful in determining whether a given high rank mixed state is entangled or not in this case, provided that we know how to extract information from Theorem 9'.

\section{Simulation of Hamiltonians}

Historically, the idea of simulating Hamiltonian (self-adjoint operators on the Hilbert space corresponding to the quantum system, see [39]) time evolutions was the first motivation for quantum computation because of the famous paper of Feynman. Recently the ability of nonlocal Hamiltonians to simulate one another is a popular topic, which has applications in quantum control theory, quantum compuation and the task of generating enatnglement. For the general treatment of this topic and the references, we refer to [9].

We say, for two bipartite Hamiltonians $H$ and $H^{\prime}$ on $H_{A}^{m} \otimes H_{B}^{n}, H^{\prime}$ can be efficiently simulated by $H$ with local unitary operations, write as $H^{\prime} \prec_{L U} H$, if $H^{\prime}$ can be written as a convex combination of conjugates of $H$ by local unitary operations, $H^{\prime}=p_{1}\left(U_{1} \otimes V_{1}\right) H\left(U_{1} \otimes V_{1}\right)^{\dagger}+\ldots+p_{s}\left(U_{s} \otimes V_{s}\right) H\left(U_{s} \otimes V_{s}\right)^{\dagger}$, where $p_{1}, \ldots, p_{s}$ are positive real numbers such that $p_{1}+\ldots+p_{s}=1, U_{1}, \ldots, U_{s}$ and $V_{1}, \ldots, V_{s}$ are unitary operations on $H_{A}^{m}$ and $H_{B}^{n}$ respectively. Here we use $\dagger$ for the adjoint. This is equivalent to the notion "infinitesimal simulation" in [9]. In [9] it is shown that "local terms" like $I \otimes K_{B}$ and $K_{A} \otimes I$ are irrelevant to the simulation problem up to the second order, thus they considered the simulation problem for Hamiltonians without local terms' effect. Our definition here is more restricted without neglecting the local terms.

We can have the following necessary conditions about the simulation of semi- 
positive Hamiltonians based on the algebraic set invariants introduced in section 2 .

Theorem 10. Let $H$ and $H^{\prime}$ be two semi-positive Hamiltonians in the bipartite quantum system $H_{A}^{m} \otimes H_{B}^{n}$. Suppose $H^{\prime} \prec_{L U} H$, that is, $H^{\prime}$ can be simulated by $H$ efficiently by using local unitary transformations. Then there are projective isomorphisms $U_{1}$ of $C P^{m-1}$ and $V_{1}$ of $C P^{n-1}$ such that $U_{1}\left(V_{A}^{k}\left(H^{\prime}\right)\right) \subset V_{A}^{k}(H)$ for $k=0, \ldots, n-1$ and $V_{1}\left(V_{B}^{k}\left(H^{\prime}\right)\right) \subset V_{B}^{k}(H)$ for $k=0, \ldots, m-1$.

The following observation about the computation of $V_{A}^{k}(\rho)$ is the the key point of the proof of Theorem 10 and Corollary 3. From Corollary 1 if $\rho=$ $\Sigma_{i}^{t} p_{i} P_{v_{i}}$ with $p_{i}$ 's positive real numbers, the range of $\rho$ is the linear span of vectors $v_{1}, \ldots, v_{t}$. We take some vectors in the set $\left\{v_{1}, \ldots, v_{t}\right\}$, say they are $v_{1}, \ldots, v_{s}$. Let $B$ be the $m n \times s$ matrix with columns corresponding to the $s$ vectors $v_{1}, \ldots, v_{s}$ 's coordinates in the standard basis of $H_{A}^{m} \otimes H_{B}^{n}$. We consider $B$ as $m \times 1$ blocked matrix with blocks $B_{1}, \ldots, B_{m} n \times s$ matrix as in section 2. It is clear that $V_{A}^{k}(\rho)$ is an algebraic subset of the zero locus of the determinants of all $(k+1) \times(k+1)$ sub-matrices of $\Sigma_{i}^{m} r_{i} B_{i}$, since $\Sigma_{i}^{m} r_{i} B_{i}$ is a sub-matrix of $\Sigma_{i}^{m} r_{i} A_{i}$. On the other hand if $v_{1}, \ldots, v_{s}$ are linear independent and $\left.s=\operatorname{dim}(\operatorname{range}(\rho)), V_{A}^{k}(\rho)\right)$ is just the zero locus of the determinants of all $(k+1) \times(k+1)$ sub-matrices of $\Sigma_{i}^{m} r_{i} B_{i}$, since any column in $\Sigma_{i} r_{i} A_{i}$ is a linear combination of columns in $\Sigma_{i} r_{i} B_{i}\left(\operatorname{rank}\left(\Sigma_{i} r_{i} A_{i}\right) \leq k\right.$ is equivalent to $\left.\operatorname{rank}\left(\Sigma_{i} r_{i} B_{i}\right) \leq k\right)$.

Proof of Theorem 10. Suppose $H^{\prime} \prec_{L U} H$, then there exist positive numbers $p_{1}, \ldots, p_{s}$ and local unitary transformations $U_{1} \otimes V_{1}, \ldots, U_{s} \otimes V_{s}$, such that, $\Sigma_{i} p_{i} U_{i} \otimes V_{i} H\left(U_{i} \otimes V_{i}\right)^{\dagger}=H^{\prime}$. Let $H=\Sigma_{i}^{s} q_{i} P_{\psi_{i}}$, where $s=\operatorname{dim}(\operatorname{range}(H))$, $q_{1}, \ldots, q_{s}$ are eigenvalues of $H$ and $\psi_{1}, \ldots, \psi_{s}$ are eigenvectors of $H$. Then it is clear that $\left(U_{i} \otimes V_{i}\right) H\left(U_{i} \otimes V_{i}\right)^{\dagger}=\Sigma_{j}^{s} q_{j} P_{\left(U_{i} \otimes V_{i}\right) \psi_{j}}$ and thus $H^{\prime}=\Sigma_{i, j} p_{i} q_{j} P_{\left(U_{i} \otimes V_{i}\right) \psi_{j}}$. This is a representation of $H^{\prime}$ as a convex combination of projections. From our above observation $V_{A}^{k}\left(H^{\prime}\right)$ is an algebraic subset in $V_{A}^{k}\left(\left(U_{1} \times V_{1}\right) H\right)$ (which can be computed from vectors $\left.\left(U_{1} \otimes V_{1}\right) \psi_{1}, \ldots,\left(U_{1} \otimes V_{1}\right) \psi_{s}\right)$. Thus the conclusion follows from Theorem 1.

Corollary 3 (see [13]). Let $H$ and $H^{\prime}$ be two semi-positive Hamiltonians in the bipartite quantum system $H_{A}^{m} \otimes H_{B}^{n}$ of the same rank, i.e. $\operatorname{dim}(\operatorname{range}(H))=$ $\operatorname{dim}\left(\operatorname{range}\left(H^{\prime}\right)\right)$. Suppose $H^{\prime} \prec_{L U} H$, that is , $H^{\prime}$ can be simulated by $H$ efficiently by using local unitary transformations. Then $V_{A}^{k}\left(H^{\prime}\right)=V_{A}^{k}(H)$ for $k=0, \ldots, n-1$ and $V_{B}^{k}\left(H^{\prime}\right)=V_{B}^{k}(H)$ for $k=0, \ldots, m-1$. Here the equality of the algebraic sets means they are isomorphic via projective linear transformations of complex projective spaces.

Proof. Suppose $H^{\prime} \prec_{L U} H$, then there exist positive numbers $p_{1}, \ldots, p_{s}$ and local unitary transformations $U_{1} \otimes V_{1}, \ldots, U_{s} \otimes V_{s}$, such that, $\Sigma_{i} p_{i} U_{i} \otimes$ $V_{i} H\left(U_{i} \otimes V_{i}\right)^{\dagger}=H^{\prime}$. Let $H=\Sigma_{i}^{s} q_{i} P_{\psi_{i}}$, where $s=\operatorname{dim}(\operatorname{range}(H)), q_{1}, \ldots, q_{s}$ are eigenvalues of $H$ and $\psi_{1}, \ldots, \psi_{s}$ are eigenvectors of $H$. Then it is clear that 
$\left(U_{i} \otimes V_{i}\right) H\left(U_{i} \otimes V_{i}\right)^{\dagger}=\Sigma_{j}^{s} q_{j} P_{\left(U_{i} \otimes V_{i}\right) \psi_{j}}$ and thus $H^{\prime}=\Sigma_{i, j} p_{i} q_{j} P_{\left(U_{i} \otimes V_{i}\right) \psi_{j}}$. This is a representation of $H^{\prime}$ as a convex combination of projections. From our above observation $V_{A}^{k}\left(H^{\prime}\right)$ can be computed from vectors $\left(U_{1} \otimes V_{1}\right) \psi_{1}, \ldots,\left(U_{1} \otimes V_{1}\right) \psi_{s}$ , since they are linear independent and $s=\operatorname{dim}\left(\operatorname{range}\left(H^{\prime}\right)\right)$. Hence $V_{A}^{k}\left(H^{\prime}\right)=$ $V_{A}^{k}\left(\left(U_{1} \otimes V_{1}\right) H\right)$ from the definition. Thus the conclusion follows from Theorem 1.

Let $S$ be the swap operator on the bipartite system $H_{A}^{n} \otimes H_{B}^{n}$ defined by $S|i j\rangle=|j i\rangle$. For any Hamiltonian $H, S(H)=S H S^{\dagger}$ corresponds to the Hamiltonian evolution of $H$ with $\mathrm{A}$ and $\mathrm{B}$ interchanged. It is very interesting to consider the problem if $H$ can be simulated by $S(H)$ efficiently. This led to some important consequences in the discussion VII of [9]. For example it was shown there are examples that $H$ and $S(H)$ cannot be simulated efficiently with one another in higher dimensions. Thus in higher dimensions nonlocal degrees of freedom of Hamiltonians cannot be characterized by quantities that are symmetric with respect to A and B, such as eigenvalues. This conclusion is also obtained from the our example and Corollary 5 in the next section. From Corollary 3 we have the following necessary condition about $H \prec_{L U} S(H)$.

Corollary 4. Let $H$ be a semi-positive Hamiltonian on $H_{A}^{n} \otimes H_{B}^{n}$. Suppose $H \prec_{L U} S(H)$. Then $V_{A}^{k}(H)=V_{B}^{k}(H)$ for $k=0, \ldots, n-1$.

The following is a Hamiltonian $H$ on $3 \times 3$ system for which $H$ cannot be simulated efficiently by $S(H)$.

Example 11. $H=P_{\left|\phi_{1}\right\rangle}+P_{\left|\phi_{2}\right\rangle}+P_{\left|\phi_{3}\right\rangle}$, where

$$
\begin{aligned}
\left|\phi_{1}\right\rangle & =\frac{1}{\sqrt{3}}(|11\rangle+|21\rangle+|32\rangle) \\
\left|\phi_{2}\right\rangle & =\frac{1}{\sqrt{1+|v|^{2}}}(|12\rangle+v|22\rangle) \\
\left|\phi_{3}\right\rangle & =\frac{1}{\sqrt{1+|\lambda|^{2}}}(|13\rangle+\lambda|23\rangle)
\end{aligned}
$$

Then it is easy to compute that $V_{A}^{2}(H)$ is the sum of 3 lines in $C P^{2}$ defined by $r_{1}+r_{2}=0, r_{1}+v r_{2}=0$ and $r_{1}+\lambda r_{2}=0$ for $v \neq \lambda$ and both $v, \lambda$ are not 1 , and $V_{B}^{2}(H)$ is the sum of 2 lines in $C P^{2}$ defined by $r_{2}=0$ and $r_{3}=0$. Thus we cannot have $H \prec_{L U} S(H)$.

\section{A continuous family of states and Hamiltoni- ans related to elliptic curves}

From physical point of view, it is very interesting to have isospectral (i.e., eigenvalues of $\rho, \operatorname{tr}_{A}(\rho), \operatorname{tr}_{B}(\rho)$ are the same) mixed states, but they are not equivalent under local unitary transformations. This phenomenon indicates that we cannot obetain a complete understanding of a bipartite quantum system by just studying the local and global properties of the spectra of the system. Some 
examples of such mixed states have been found by several authors(see Nielsen and Kempe [37] and references there). Here we give a continuous family of such mixed states.

Let $H=H_{A}^{3} \otimes H_{B}^{3}$ and $\rho_{\eta_{1}, \eta_{2}, \eta_{3}}=\frac{1}{3}\left(P_{\left|v_{1}\right\rangle}+P_{\left|v_{2}\right\rangle}+P_{\left|v_{3}\right\rangle}\right)\left(\eta_{1}, \eta_{2}, \eta_{3}\right.$ are real parameters), a continuous family of mixed states on $H$, where

$$
\begin{aligned}
& \left|v_{1}\right\rangle=\frac{1}{\sqrt{3}}\left(e^{i \eta_{1}}|11\rangle+|22\rangle+|33\rangle\right) \\
& \left|v_{2}\right\rangle=\frac{1}{\sqrt{3}}\left(e^{i \eta_{2}}|12\rangle+|23\rangle+|31\rangle\right) \\
& \left.\left|v_{3}\right\rangle=\frac{1}{\sqrt{3}}\left(e^{i \eta_{3}}|13\rangle+|21\rangle+32\right\rangle\right)
\end{aligned}
$$

It is easy to calculate that $\Sigma r_{i} A_{i}$ (up to a constant) is the following $3 \times 3$ matrix

$$
\left(\begin{array}{ccc}
e^{i \eta_{1}} r_{1} & r_{3} & r_{2} \\
r_{2} & e^{i \eta_{2}} r_{1} & r_{3} \\
r_{3} & r_{2} & e^{i \eta_{3}} r_{1}
\end{array}\right)
$$

Thus $V_{A}^{2}\left(\rho_{\eta_{1}, \eta_{2}, \eta_{3}}\right)$ is defined by $e^{i\left(\eta_{1}+\eta_{2}+\eta_{3}\right)} r_{1}^{3}+r_{2}^{3}+r_{3}^{3}-\left(e^{i \eta_{1}}+e^{i \eta_{2}}+\right.$ $\left.e^{i \eta_{3}}\right) r_{1} r_{2} r_{3}=0$ in $C P^{2}$. With $e^{i\left(\eta_{1}+\eta_{2}+\eta_{3}\right) / 3} r_{1}=r_{1}^{\prime}$ we have $r_{1}^{\prime 3}+r_{2}^{3}+r_{3}^{3}-$ $\frac{e^{i \eta_{1}}+e^{i \eta_{2}}+e^{i \eta_{3}}}{e^{i\left(\eta_{1}+\eta_{2}+\eta_{3}\right) / 3}} r_{1}^{\prime} r_{2} r_{3}=0$. This is a family of elliptic curves.

It is easy to check that 3 nonzero eigenvalues of $\rho_{\eta_{1}, \eta_{2}, \eta_{3}}, \operatorname{tr}_{A}\left(\rho_{\eta_{1}, \eta_{2}, \eta_{3}}\right), \operatorname{tr}_{B}\left(\rho_{\eta_{1}, \eta_{2}, \eta_{3}}\right)$ are all the same value $\frac{1}{3}$ for different parameters. In this case we have a family of isospectral (both global and local) mixed states $\rho_{\eta_{1}, \eta_{2}, \eta_{3}}$. Set $g\left(\eta_{1}, \eta_{2}, \eta_{3}\right)=$

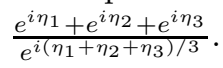

Theorem 11. $\rho_{\eta_{1}, \eta_{2}, \eta_{3}}$ is entangled mixed state when $\left(g\left(\eta_{1}, \eta_{2}, \eta_{3}\right)\right)^{3} \neq$ $0,-216,27$. Moreover $\rho_{\eta_{1}, \eta_{2}, \eta_{3}}$ and $\rho_{\eta_{1}^{\prime}, \eta_{2}^{\prime}, \eta_{3}^{\prime}}$ are not equivalent under local unitary transformations if $k\left(g\left(\eta_{1}, \eta_{2}, \eta_{3}\right)\right) \neq k\left(g\left(\eta_{1}^{\prime}, \eta_{2}^{\prime}, \eta_{3}^{\prime}\right)\right)$, where $k(x)=\frac{x^{3}\left(x^{3}+216\right)^{3}}{\left(-x^{3}+27\right)^{3}}$ is the moduli function of elliptic curves.

Proof. The conclusion follows from Theorem 3,1 and the well-known fact about elliptic curves (see [10])

From Theorem 11 we give continuous many isospectral non-local-equivalent rank 3 mixed states in $H$.

In [36] Nielsen gave a beautiful necessary and sufficient condition for the bipartite pure state $|\psi\rangle$ can be transformed to the pure state $|\phi\rangle$ by local operations and classical communications (LOCC) based on the majorization between the eigenvalue vectors of the partial traces of $|\psi\rangle$ and $|\phi\rangle$. In [8] an example was given, from which we know that Nielsen's criterion cannot be generalized to multipartite case, $\mathbf{3 E P R}$ and $\mathbf{2 G H Z}$ are understood as pure states in a $4 \times 4 \times 4$ 
quantum system, they have the same eigenvalue vectors when traced over any subsystem. However it is proved that they are LOCC-incomparable in [8]

In the following example, a continuous family $\left|\{\phi\}_{\eta_{1}, \eta_{2}, \eta_{3}}\right\rangle$ of pure states in tripartite quantum system $H_{A_{1}}^{3} \otimes H_{A_{2}}^{3} \otimes H_{A_{3}}^{3}$ is given, the eigenvalue vectors of $\operatorname{tr}_{A_{i}}\left(P_{\left|\phi_{\eta_{1}, \eta_{2}, \eta_{3}}\right\rangle}\right), \operatorname{tr}_{A_{i} A_{j}}\left(P_{\left|\phi_{\eta_{1}, \eta_{2}, \eta_{3}}\right\rangle}\right)$ are independent of parameters $\eta_{1}, \eta_{2}, \eta_{3}$. However the generic pure states in this family are entangled and LOCC-incomparable. This gives stronger evidence that it is hopeless to characterize the entanglement properties of multipartite pure states by only using the eigenvalue spetra of their partial traces.

Let $H=H_{A_{1}}^{3} \otimes H_{A_{2}}^{3} \otimes H_{A_{3}}^{3}$ be a tripartite quantum system and $\left|\phi_{\eta_{1}, \eta_{2}, \eta_{3}}\right\rangle=$ $\frac{1}{\sqrt{3}}\left(\left|v_{1}\right\rangle \otimes|1\rangle+\left|v_{2}\right\rangle \otimes|2\rangle+\left|v_{3}\right\rangle \otimes|3\rangle\right)$, where $\left|v_{1}\right\rangle,\left|v_{2}\right\rangle,\left|v_{3}\right\rangle$ are as in (19). This is a continuous family of pure states in $H$ parameterized by three real parameters. We can check that the eigenvalue vector of any partial trace of $P_{\left|\phi_{\eta_{1}, \eta_{2}, \eta_{3}}\right\rangle}$ is a constant vector. On the other hand it is clear that $\operatorname{tr}_{A_{3}}\left(P_{\left|\phi_{\eta_{1}, \eta_{2}, \eta_{3}}\right\rangle}\right)=\frac{1}{3}\left(P_{\left|v_{1}\right\rangle}+\right.$ $\left.P_{\left|v_{2}\right\rangle}+P_{\left|v_{3}\right\rangle}\right)$ is a rank 3 mixed state in $H_{A_{1}}^{3} \otimes H_{A_{2}}^{3} \cdot\left|\phi_{\eta_{1}, \eta_{2}, \eta_{3}}\right\rangle$ and $\left|\phi_{\eta_{1}^{\prime}, \eta_{2}^{\prime}, \eta_{3}^{\prime}}\right\rangle$ are not equivalent under local unitary transformations if $k\left(g\left(\eta_{1}, \eta_{2}, \eta_{3}\right)\right) \neq k\left(g\left(\eta_{1}^{\prime}, \eta_{2}^{\prime}, \eta_{3}^{\prime}\right)\right)$, since their corresponding traces over $A_{3}$ are not equivalent under local unitary transformations of $H_{A_{1}}^{3} \otimes H_{A_{2}}^{3}$ from Theorem 11. Hence the generic members of this family of pure states in tripartite quantum system $H$ are enatngled and LOCC-incomparable from Theorem 1 in [8].

We can also consider the following continuous family of semi-positive Hamiltonians depending on 3 real parameters, $H_{\eta_{1}, \eta_{2}, \eta_{3}}=P_{\left|v_{1}\right\rangle}+P_{\left|v_{2}\right\rangle}+P_{\left|v_{3}\right\rangle}$, where $v_{1}, v_{2}, v_{3}$ are as in (19). As calculated above, $V_{A}^{2}\left(H_{\eta_{1}, \eta_{2}, \eta_{3}}\right)$ is just the elliptic curve in $C P^{2}$ defined by $r_{1}^{3}+r_{2}^{3}+r_{3}^{3}-\frac{e^{i \eta_{1}}+e^{i \eta_{2}}+e^{i \eta_{3}}}{e^{i\left(\eta_{1}+\eta_{2}+\eta_{3}\right) / 3}} r_{1} r_{2} r_{3}=0$. The elliptic curve $V_{A}^{2}\left(H_{\eta_{1}, \eta_{2}, \eta_{3}}\right)$ is not isomorphic to the elliptic curve $V_{A}^{2}\left(H_{\eta_{1}^{\prime}, \eta_{2}^{\prime}, \eta_{3}^{\prime}}\right)$ if $k\left(g\left(\eta_{1}, \eta_{2}, \eta_{3}\right)\right) \neq k\left(g\left(\eta_{1}^{\prime}, \eta_{2}^{\prime}, \eta_{3}^{\prime}\right)\right)$. Thus we have the following Corollary of Theorem 9 .

Corollary 5. $H_{\eta_{1}^{\prime}, \eta_{2}^{\prime}, \eta_{3}^{\prime}}$ cannot be simulated by $H_{\eta_{1}, \eta_{2}, \eta_{3}}$ efficiently by using local unitary transformations,i.e., we cannot have $H_{\eta_{1}^{\prime}, \eta_{2}^{\prime}, \eta_{3}^{\prime}} \prec_{L U} H_{\eta_{1}, \eta_{2}, \eta_{3}}$, if $k\left(g\left(\eta_{1}, \eta_{2}, \eta_{3}\right)\right) \neq k\left(g\left(\eta_{1}^{\prime}, \eta_{2}^{\prime}, \eta_{3}^{\prime}\right)\right)$, though the 3 nonzero eigenvalues of $H_{\eta_{1}, \eta_{2}, \eta_{3}}$, $H_{\eta_{1}^{\prime}, \eta_{2}^{\prime}, \eta_{3}^{\prime}}$ and their partial traces are all 1 .

\section{Constructing entangled PPT mixed states}

As mentioned in Introduction, the first several entangled PPT mixed states were constructed in [25] based on P. Horodecki's range criterion of separable states, which asserts that a separable mixed state has to include sufficiently many separable pure states in its own range (see [25],[28]). This range criterion of separable mixed states was also the base to construct PPT entangled 
mixed states in the context of unextendible product base (UPB) studied by C.H.Bennett, D.P.DiVincenzo, T.Mor, P.Shor, J.A.Smolin and T.M.Terhal in [7] (We should mention that unextendible product base also have other physical significance nonlocality without entanglement, see [7],[28]). It is always interesting and important to have more methods to construct entangled PPT mixed states. In this section, we give an example to show how our separability criterion Theorem 3 can be used to construct entangled mixed states which are invariant under partial transposition (thus PPT and bound entanglement) systematically.

In the following example we construct a family of rank 7 mixed states $\left\{\rho_{e_{1}, e_{2}, e_{3}}\right\}\left(e_{1}, e_{2}, e_{3}\right.$ are real parameters) with $\rho_{e_{1}, e_{2}, e_{3}}=\rho_{e_{1}, e_{2}, e_{3}}^{P T}$ (hence PPT automatically) on $H=H_{A}^{4} \otimes H_{B}^{6}$. We prove that they are entangled by Theorem 3 (thus bound entanglement) for generic parameters $e_{1}, e_{2}, e_{3}$ and parameters $\left(e_{1}, e_{2}, e_{3}\right)=(0,0,1)$. This family and the method used here can be easily generalized to construct entangled mixed states with $\rho=\rho^{P T}$ systematically.

Consider the following $46 \times 7$ matrices

$$
\begin{gathered}
A_{1}=\left(\begin{array}{ccccccc}
1 & 0 & 0 & 0 & 0 & 0 & 0 \\
0 & 1 & 0 & 0 & 0 & 0 & 0 \\
0 & 0 & 1 & 0 & 0 & 0 & 0 \\
0 & 0 & 0 & 2 & 0 & 0 & 1 \\
0 & 0 & 0 & 0 & 2 & 0 & 0 \\
0 & 0 & 0 & 0 & 0 & 2 & 0
\end{array}\right) \\
A_{3}=\left(\begin{array}{ccccccc}
0 & 1 & 1 & -1 & 0 & 0 & 1 \\
1 & 0 & 1 & 0 & 0 & 0 & 0 \\
1 & 1 & 0 & 0 & 0 & 0 & 0 \\
-1 & 0 & 0 & 0 & 1 & 1 & 0 \\
0 & 0 & 0 & 1 & 0 & 1 & 0 \\
0 & 0 & 0 & 1 & 1 & 0 & 0
\end{array}\right) \\
\left(\begin{array}{cccccccc}
A_{2}+e_{3} & e_{1} & 0 & & 0 & 0 & 0 & 0 \\
e_{1} & e_{2} & e_{3} & 0 & 0 & 0 & 0 \\
0 & e_{3} & e_{1}+e_{2} & 0 & 0 & 0 & 0 \\
0 & 0 & 0 & e_{2}+e_{3} & e_{1} & 0 & 0 \\
0 & 0 & 0 & e_{1} & e_{2} & e_{3} & 0 \\
0 & 0 & 0 & 0 & e_{3} & e_{1}+e_{2} & 0
\end{array}\right)
\end{gathered}
$$

, where $e_{1}, e_{2}, e_{3}$ are real parameters, and $A_{4}=\left(I_{6}, 0\right)$, where $I_{6}$ is $6 \times 6$ unit matrix.

Let $A$ be a $24 \times 7$ matrix with 4 blocks $A_{1}, A_{2}, A_{3}, A_{4}$ where the 24 rows correspond to the standard basis $\{|11\rangle, \ldots,|16\rangle, \ldots,|41\rangle, \ldots,|46\rangle\}$. Let $\rho_{e_{1}, e_{2}, e_{3}}$ be $\frac{1}{D} A A^{\dagger}$ (where $D$ is a normalizing constant), a mixed state on $H$. It is easy to check that $A_{i} A_{j}^{\dagger}=A_{j} A_{i}^{\dagger}$, hence $\rho_{e_{1}, e_{2}, e_{3}}$ is invariant under partial transposition 
and thus PPT.

Let $\left|\psi_{1}\right\rangle, \ldots,\left|\psi_{7}\right\rangle \in H_{A}^{4} \otimes H_{B}^{6}$ be 7 vectors corresponding to 7 columns of the matrix $A$. It is clear that the range of $\rho_{e_{1}, e_{2}, e_{3}}$ is the linear span of $\left|\psi_{1}\right\rangle, \ldots,\left|\psi_{7}\right\rangle$. When $e_{1}=e_{2}=0, e_{3}=1,\left|\psi_{2}\right\rangle-\left|\psi_{3}\right\rangle=(|1\rangle+|4\rangle-|2\rangle) \otimes(|2\rangle-|3\rangle)$. Thus there are some separable pure states in the range of $\rho_{0,0,1}$. We will show that $\rho_{0,0,1}$ and $\rho_{e_{1}, e_{2}, e_{3}}$ for generic parameters $e_{1}, e_{2}, e_{3}$ are entangled by our separability criterion Theorem 3 .

As in the proof of Theorem 2 it is easy to compute $F=r_{1} A_{1}+r_{2} A_{2}+r_{3} A_{3}+$ $r_{4} A_{4}$.

$$
\left(\begin{array}{ccccccc}
u_{1} & r_{2}+e_{1} r_{3} & r_{2} & -r_{2} & 0 & 0 & r_{2} \\
r_{2}+e_{1} r_{3} & u_{1}^{\prime} & r_{2}+e_{3} r_{3} & 0 & 0 & 0 & 0 \\
r_{2} & r_{2}+e_{3} r_{3} & u_{1}^{\prime \prime} & 0 & 0 & 0 & 0 \\
-r_{2} & 0 & 0 & u_{2} & r_{2}+e_{1} r_{3} & r_{2} & r_{1} \\
0 & 0 & 0 & r_{2}+e_{1} r_{3} & u_{2}^{\prime} & r_{2}+e_{3} r_{3} & 0 \\
0 & 0 & 0 & r_{2} & r_{2}+e_{3} r_{3} & u_{2}^{\prime \prime} & 0
\end{array}\right)
$$

, where $u_{1}=r_{1}+r_{4}+\left(e_{2}+e_{3}\right) r_{3}, u_{1}^{\prime}=r_{1}+r_{4}+e_{2} r_{3}, u_{1}^{\prime \prime}=r_{1}+r_{4}+\left(e_{1}+e_{2}\right) r_{3}$ and $u_{2}=2 r_{1}+r_{4}+\left(e_{2}+e_{3}\right) r_{3}, u_{2}^{\prime}=2 r_{1}+r_{4}+e_{2} r_{3}, u_{2}^{\prime \prime}=2 r_{1}+r_{4}+\left(e_{1}+e_{2}\right) r_{3}$.

We consider the following matrix $F^{\prime}$ which is obtained by adding the 7 -th column of $F$ to the 4-th column of $F$ and adding $r_{2} / r_{1}$ of the 7 -th column to the 1 st column.

$$
\left(\begin{array}{ccccccc}
v_{1} & r_{2}+e_{1} r_{3} & r_{2} & 0 & 0 & 0 & r_{2} \\
r_{2}+e_{1} r_{3} & u_{1}^{\prime} & r_{2}+e_{3} r_{3} & 0 & 0 & 0 & 0 \\
r_{2} & r_{2}+e_{3} r_{3} & u_{1}^{\prime \prime} & 0 & 0 & 0 & 0 \\
0 & 0 & 0 & v_{2} & r_{2}+e_{1} r_{3} & r_{2} & r_{1} \\
0 & 0 & 0 & r_{2}+e_{1} r_{3} & u_{2}^{\prime} & r_{2}+e_{3} r_{3} & 0 \\
0 & 0 & 0 & r_{2} & r_{2}+e_{3} r_{3} & u_{2}^{\prime \prime} & 0
\end{array}\right)
$$

, where $v_{1}=r_{1}+r_{4}+\left(e_{2}+e_{3}\right) r_{3}+\frac{r_{2}^{2}}{r_{1}}, v_{2}=3 r_{1}+r_{4}+\left(e_{2}+e_{3}\right) r_{3}$.

It is clear that the determinantal varieties defined by $F$ and $F^{\prime}$ are the same in the affine chart $C^{3}$ defined by $r_{1} \neq 0$. Consider the zero locus $Z_{1}$ defined by the condition that the determinants of the 2 diagonal $3 \times 3$ submtrices of the 1 st $6 \times 6$ submatrix in $(21)$ are zero, locus $Z_{2}$ defined by the condition that the 1st 3 rows in (21) are linear dependent and the locus $Z_{3}$ defined by the condition that the last 3 rows in (21) are linear dependent, it is clear that $V_{A}^{5}\left(\rho_{e_{1}, e_{2}, e_{3}}\right) \cap C^{3}$ is the sum of $Z_{1}, Z_{2}, Z_{3}$. We can use the affine coordinates $r_{2}^{\prime}=\frac{r_{2}}{r_{1}}, r_{3}^{\prime}=\frac{r_{3}}{r_{1}}, r_{4}^{\prime}=\frac{r_{4}}{r_{1}}$ on the affine chart $C^{3}$ of $C P^{3}$ defined by $r_{1} \neq 0$. In this 
affine coordinate system all $r_{2}, r_{3}, r_{4}$ should be replced by $r_{2}^{\prime}, r_{3}^{\prime}, r_{4}^{\prime}$ and $r_{1}$ should be replaced by 1 in (21). Now we analysis $V_{A}^{5}\left(\rho_{0,0,1}\right)$. It is clear that the following 2 planes $H_{1}=\left\{\left(r_{2}^{\prime}, r_{3}^{\prime}, r_{4}^{\prime}\right): r_{2}^{\prime}=r_{4}^{\prime}+1\right\}, H_{2}=\left\{\left(r_{2}^{\prime}, r_{3}^{\prime}, r_{4}^{\prime}\right): r_{2}^{\prime}=r_{4}^{\prime}+2\right\}$ are in $V_{A}^{5}\left(\rho_{0,0,1}\right) \cap C^{3}$, since in the case $r_{2}^{\prime}=r_{4}^{\prime}+1$ the 2 nd and the 3 rd rows of $(21)$ are linearly dependent and in the case $r_{2}^{\prime}=r_{4}^{\prime}+2$ the 5 th and 6 th rows of $(21)$ are linearly dependent. The determinants of two $3 \times 3$ diagonal submatrices of the 1 st $6 \times 6$ submatrix of $(21)$ are:

$$
\begin{gathered}
\left(r_{2}^{\prime}-r_{4}^{\prime}-1\right)\left(\left(r_{2}^{\prime}\right)^{3}+\left(r_{2}^{\prime}\right)^{2} r_{4}^{\prime}-\left(r_{2}^{\prime}\right)^{2}+\left(r_{4}^{\prime}\right)^{2}\right. \\
\left.+r_{2}^{\prime} r_{3}^{\prime}+r_{2}^{\prime} r_{4}^{\prime}+r_{3}^{\prime} r_{4}^{\prime}+r_{2}^{\prime}+r_{3}^{\prime}+2 r_{4}^{\prime}+1\right)
\end{gathered}
$$

and

$$
\left(r_{2}^{\prime}-r_{4}^{\prime}-2\right)\left(\left(r_{4}^{\prime}\right)^{2}-2\left(r_{2}^{\prime}\right)^{2}+r_{2}^{\prime} r_{3}^{\prime}+r_{2}^{\prime} r_{4}^{\prime}+r_{3}^{\prime} r_{4}^{\prime}+3 r_{2}^{\prime}+2 r_{3}^{\prime}+5 r_{4}^{\prime}+6\right)
$$

Let $X_{1}$ and $X_{2}$ be zero locus of the 2 nd factors of the above two determinants. It is obvious $X_{1} \cap X_{2}$ is in $V_{A}^{5}\left(\rho_{0,0,1}\right) \cap C^{3}$, we want to show that $X_{1} \cap X_{2} \backslash H_{1} \cup H_{2}$ is a curve, not a line. Take the ponit $P=(0,2,-1) \in X_{1} \cap X_{2} \cap H_{1}$, the tangent plane $H_{3}$ of $X_{2}$ at $P$ is defined by $4 r_{2}^{\prime}+r_{3}^{\prime}+5 r_{4}^{\prime}=-3$. If $X_{1} \cap X_{2}$ is a line around the ponit $P$, this line is contained in $H_{3} \cap X_{2}$. However we can easily find that $H_{3} \cap X_{2}$ is defined by $3\left(r_{2}^{\prime}\right)^{2}+2\left(r_{4}^{\prime}\right)^{2}+4 r_{2}^{\prime} r_{4}^{\prime}+4 r_{4}^{\prime}=0$. This polynomial is irreducible and thus $H_{3} \cap X_{2}$ is a curve around the piont $P$. Thus $X_{1} \cap X_{2}$ is a curve around the point $P$. It is easy to check that $X_{1} \cap X_{2}$ is not contained in $H_{1}$ around the point $P$. This implies that $V_{A}^{5}\left(\rho_{e_{1}, e_{2}, e_{3}}\right) \cap C^{3}$ (actually the locus $Z_{1}$ ) contains a curve (not a line) for generic parameters $e_{1}, e_{2}, e_{3}$ (including parameters $0,0,1)$ from algebraic geometry. Thus if $V_{A}^{5}\left(\rho_{e_{1}, e_{2}, e_{3}}\right) \cap C^{3}$ is the sum of (affine) linear subspaces, it have to contain a dimension 2 affice linear subspace $H_{4}$ other than $H_{1}$ and $H_{2}$ of the affine chart $C^{3}$. Thus the determinants of all $6 \times 6$ submatrices of (21) have to contain an (fixed) affine linear form (i.e., a degree one polynomial of $r_{2}^{\prime}, r_{3}^{\prime}, r_{4}^{\prime}$ which may contain a constant term) other than $r_{2}^{\prime}-r_{4}^{\prime}-1$ and $r_{2}^{\prime}-r_{4}^{\prime}-1$ as one of their factors. This affine linear form defines that dimension 2 linear affine subspace $H_{4}$ of $C^{3}$. However it is easy to check this is impossible for generic parameters $e_{1}, e_{2}, e_{3}$ (including parameters $0,0,1)$. We know that $V_{A}^{5}\left(\rho_{e_{1}, e_{2}, e_{3}}\right) \cap C^{3}$ cannot be the sum of (affine) linear subspaces of $C^{3}$ for generic $e_{1}, e_{2}, e_{3}$ (including parameters $0,0,1$ ). Thus from Theorem $3 \rho_{e_{1}, e_{2}, e_{3}}$ is entangled for generic parameters $e_{1}, e_{2}, e_{3}$ (including parameters $0,0,1$ ).

Theorem 12. The mixed states $\rho_{e_{1}, e_{2}, e_{3}}$ 's, which are invariant under partial transposition, are entangled for generic parameters and $\left(e_{1}, e_{2}, e_{3}\right)=(0,0,1)$.

Remark 4. $\rho_{0,0,1}$ is the first example of PPT entangled mixed state (thus bound entanglement) with some separable pure states in its range.

From the construction in this Example we can see if $A_{1}, \ldots, A_{m}$ are $m n \times t$ matrices satisfying $A_{i} A_{j}^{\dagger}=A_{j} A_{i}^{\dagger}, A$ is the $m \times 1$ matrix with i-th block $A_{i}$ 
and the rows of $A$ correspond to the basis $|11\rangle, \ldots,|1 n\rangle, \ldots,|m 1\rangle, \ldots,|m n\rangle$ of $H_{A}^{m} \otimes H_{B}^{n}$, then the mixed state $\rho=\frac{1}{D} A A^{\dagger}$, where $D$ is a normalized constant, is invariant under partial transpose. It is not very difficult to find such matrices. For the purpose that the constructed mixed state $\rho$ is entangled (thus a bound entangled mixed state), we just need that the determinantal variety $\left\{\left(r_{1}, \ldots, r_{m}\right): \operatorname{rank}\left(\Sigma r_{i} A_{i}\right) \leq n-1\right\}$ is NOT linear. We know from algebraicgeometry, it is not very hard to find such matrices $A_{1}, \ldots, A_{m}$. However as illustrated in this Example we do need some explicit calculation to prove this point. Thus our separability criterion and the method used in this Example offer a new systematic way to construct PPT bound entangled mixed states.

Acknowledgment. This work was supported by NNSF China grant 10171110 and NNSF China Distinguished Young Scholar Grant 10225106.

\section{REFERENCES}

1.S.Abhyankar, Combinatorics of Young tableaux, determinantal varieties and the calculation of Hilbert functions(in French), Rend. Sem. Mat. Univ. Politec. Torino, 42(1984), no.3, pp65-88

2.A.Acin, A.Andrianov, L.Costa, E.Jane, J.I.Latorre and R.Tarrach, Generalized Schmidt decomposition and classification of three-qubit states, Phys. Rev. Lett. 85, 1560,2001

3.A.Acin, D.Bruss, M.Lewenstein and A.Sanpera, Classification mixed threequbit states, Phys. Rev. Lett. 87, 040401(2001)

4.E.Arbarello, M.Cornalba, P.A.Griffiths and J.Harris, Geometry of algebraic curves, Volume I, Springer-Verlag, 1985, Chapter II Determinantal Varieties

5.C.H. Bennett and P.W.Shor, Quantum Information Theory, IEEE Trans. Inform. Theory, vol.44(1998), No.6

6.C.H.Bennett, G.Brassard, C.Crepeau, R.Jozsa, A.Peres and W.K.Wootters, Teleporting an unknown quantum state via dual classical and Einstein-PodolskyRosen channels, Phys. Rev. Lett. 70, 1895 (1993)

7.C.H.Bennett, D.P.DiVincenzo, T.Mor, P.W. Shor, J.A.Smolin and T.M. Terhal, Unextendible product bases and bound entanglement, Phys. Rev. Lett. $82,5385-5388,1999$

8.C.H.Bennett, S.Popescu, D.Rohrlich, J.A.Smolin and A.Thapliyal, Exact and asymptotic measure of multipartite pure-state entanglement, Phys. Rev. A 63, 012307, 2000 
9.C.H.Bennett, J.I.Cirac, M.S.Leifer, D.W.Leung, N.Linden, S.Popescu and G.Vidal, Optimal simulation of two-qubit Hamiltonians using general local operations, quant-ph/0107035. Phys.Rev. A, 66, 012305(2002)

10.E.Brieskorn and H.Knorrer, Plane algebraic curves, Birkhauser Boston,1981

11.J-L. Brylinski and R. Brylinski, Invariant polynomial functins on $\mathrm{k} q u-$ dits, Mathematics of Quantum Computation, edited by R.Brylinski and G. Chen CRC Press, 2002

12.J-L. Brylinski and R.Brylinski, Universal quantum gates, Mathematics of Quantum Computation, edited by R.Brylinski and G. Chen, CRC Press, 2002

13.H. Chen, Necessary conditions for efficient simulation of Hamiltonians using local unitary operations, quant-ph/0109115, Quantum Information and Computation Vol.3,No.3 (2003), pp249-257

14.H. Chen, Schmidt numbers of low rank bipartite mixed states, quant-ph/0211052 Phys. Rev. A, Vol.67(2003),062301

15.C.De Concini, D.Eisenbud and C.Procesi, Young diagrams and determinantal varieties, Invent. Math., 56(1980), no.2, pp129-165

16.A.Ekert and R.Jozsa, Quantum algorithms: entanglement enhanced information processing, Phil. Trans. Roy. Soc. (Lond.), 1998

17.A.Ekert, Quantum cryptography based on Bell's theorem, Phys Rev. Lett. 67, 661(1991)

18.A.Einstein, B.Podolsky and N.Rosen, Can quantum mechanical description of physical reality be considered complete, Phys. Rev. 47, 777(1935)

19.D.Eisenbud, J.Koh and M.Stillman, Determinantal equations for curves of high degree, Amer.J.Math., vol.110(1988),pp513-539

20.D.Eisenbud, Linear sections of determinantal varieties, Amer.J.Math., Vol.110(1988), pp.541-575

21.D.M.Greenberger, M.Horne and A.Zeilinger, Bell's Theorem, Quantum Theory, and Conceptions of the Universe, edited by M.Kafatos (Kluwer Dordrecht)

22.P.A.Griffiths, Infinitesimal variations of Hodges structure III: Determinantal varieties and the infinitesimal invariants of normal functions, Compositio Math., 50(1983), no.2-3, pp267-324 
23.J.Harris, Algebraic geometry, A first Course, Gradute Texts in Mathematics, 133, Springer-Verlag, 1992, especially Lecture 9 Determinantal Varieties

24.M.Horodecki, P.Horodecki and R.Horodecki, Separability of mixed states: Necessary and sufficient conditions, Phys. Lett. A 223, 8 (1996)

25.P.Horodecki,Separability criterion and inseparable mixed states with positive partial transpose, Phys. Lett. A 232 333(1997)

26.M.Horodecki, P.Horodecki and R.Horodecki, Mixed state entanglement and distillation: is there "bound" entanglement in nature, Phys. Rev. Lett. 80, $5239(1998)$

27.M.Horodecki, P.Horodecki and R.Horodecki, Separability of n-particle mixed states: necessary and sufficient conditios in terms of linear maps, quant-ph/0006071 Phys. Lett. A, 283(2001)

28.M.Horodecki , P.Horodecki and R. Horodecki, Mixed state entanglement and quantum communication, in "Quantum Infomation-Basic concepts and experiments", Eds, G.Alber and M.Wiener (Springer Berlin)

29.P.Horodecki, M.Lewenstein, G.Vidal and J.I. Cirac,Operational criterion and constructive checks for the separability of low rank density matrices, Phys. Rev. A 62, 032310 (2000)

30.B.Kiraus, J.I.Cirac, S.Karnas and M.Lewenstein, Separability in 2xN composite quantum systems, Phys. Rev. A 61, 062302 (2000)

31.L.H.Kauffman and S.J.Jr.Lomonaco, Quantum entanglement and topological entanglement, New Journal of Physics, 4 ,73(2002)

32.M. Lewenstein, D Bruss, J.I.Cirac, B.Krus, M.Kus, J. Samsonowitz , A.Sanpera and R.Tarrach, Separability and distillablity in composite quantum systems-a premier, J.Mod. Optics, 47, 2481(2000)

33.N.Linden and S.Popescu,On multi-particle entanglement, Fortsch. Phys.,46, $567(1998)$

34.N.Linden, S.Popescu and A.Sudbery, Non-local parameters of multi-particle density matrices, Phys. Rev. Lett, 83(1999), 243-247

35.D.Meyer and N.R.Wallach, Global entanglement in multiparticle systems, J. Math. Phys., 4273-4278, no.9, 43(2002)

36.M.A.Nielsen, Conditions for a class of entanglement transformations, Phys. Rev. Lett. 83, 436 (1999) 
37.M.A.Nielsen and J.Kempe, Separable states are more disordered globally than locally, Phys. Rev. Lett., 86, 5184 (2001)

38.A.Peres, Separability criterion for density matrices, Phys. Rev. Lett. $77,1413-1415(1996)$

39.J.Preskill, Physics 229: Advanced mathematical methods of PhysicsQuantum Computation and Information (California Institute of Technology, Pasadena,CA,1998), http://www.theory.caltech.edu/people/preskill/ph229/

40.J.A.Smolin, Four-party unlockable bound-entangled states, Phys. Rev. A $63,032306(2001)$

41.B.Terhal and P.Horodecki, Schmidt number for density matrices, Phys. Rev. A61, 040301(2000)

42.B.M.Terhal, Detecting quantum entanglements, quant-ph/0101032, Theoret. Comput. Sci., 287(2002),no.1

43.N.R.Wallach, An unentangled Gleason's theorem, Contemp. Math. 305 (2002), AMS, Providence, RI

44.R.F.Werner and M.M.Wolf, Bell's inequality for states with positive partial transpose, Phys. Rev. A 61, 062102 (2000) 